\title{
Initial Systematic Investigations of the Landscape of Low-Layer NAHE Variation Extensions
}

\author{
Timothy Renner, Jared Greenwald, Douglas Moore, and Gerald Cleaver
}

EUCOS-CASPER, Department of Physics, Baylor University, Waco, TX 76978, USA

Correspondence should be addressed to Gerald Cleaver; gerald_cleaver@baylor.edu

Received 3 June 2013; Accepted 8 July 2013

Academic Editors: C. A. D. S. Pires, A. Koshelev, and M. Masip

Copyright (C) 2013 Timothy Renner et al. This is an open access article distributed under the Creative Commons Attribution License, which permits unrestricted use, distribution, and reproduction in any medium, provided the original work is properly cited.

\begin{abstract}
The discovery that the number of physically consistent string vacua is on the order of $10^{500}$ has prompted several statistical studies of string phenomenology. Focusing on the Weakly Coupled Free Fermionic String formalism, we present systematic extensions of a variation on the NAHE (Nanopoulos, Antoniadis, Hagelin, Ellis) set of basis vectors. This variation is more conducive to the production of "mirrored" models, in which the observable and hidden sector gauge groups (and possibly matter content) are identical. This study is parallel to the extensions of the NAHE set itself and presents statistics related to similar model properties. Statistical coupling between specific gauge groups and spacetime supersymmetry is also examined. Finally, a model with completely mirrored gauge groups is discussed. It is found that the region of the landscape explored generates no physically realistic models due to a lack of three net chiral generations.
\end{abstract}

\section{Introduction}

The large number of string vacua $[1,2]$ has prompted both computational and analytical examinations of the landscape, for example, [3-11]. The Weakly Coupled Free Fermionic Heterotic String (WCFFHS) [12-15] approach to string model construction has produced some of the most phenomenologically realistic string models to date [16-57]. The present study focuses on the systematic extension of an NAHE Variation [50] thereby scanning a region the WCFFHS parameter space yet to be explored. The NAHE Variation is of particular interest because it is conducive to the generation of mirror models. Additionally, it was hoped that this regime would produce models with three net chiral generations which turns out not to be the case. Traditionally, the number of fermion families is linked to the topological structure of the compactification; however, in the language of the WCFFHS this connection is difficult to explore analytically because many such models do not have a well-defined geometric interpretation. This work parallels that presented in [58] regarding NAHE extension.

1.1. The NAHE Variation. While there have been many quasirealistic models constructed from the NAHE basis, other bases can be used to create different classes of realistic and quasirealistic heterotic string models. Like the NAHE set, the NAHE variation is a collection of five order-2 basis vectors. However, the sets of matching boundary conditions are larger than those of the NAHE set. This allows for a new class of models with "mirrored" groups, that is, with gauge groups that occur in even factors. Some also have mirrored matter representations that do not interact with one another. This means that hidden sector content matches the observable sector, making the dark matter and observable matter gauge charges identical. Several scenarios with mirrored dark matter have been presented as viable phenomenological descriptions of the universe [59-62].

The NAHE set does not have a tendency to produce mirrored models because the boundary conditions making up the $S U(4)^{3}$ gauge groups break the mirroring between the elements $\bar{\psi}, \bar{\eta}$, and $\bar{\phi}$. We can remedy this by ensuring that the worldsheet fermions $\bar{\psi}^{1, \ldots, 5}$ and $\bar{w}^{1, \ldots, 6}$ have the same boundary conditions as $\bar{\phi}^{1, \ldots, 8}$. In doing so, the NAHE variation basis vectors generate a model with gauge group $S O(22) \otimes E_{6} \otimes U(1)^{5}$. The basis vectors making up this set are presented in Table 1 with the resulting particle content of the NAHE variation model presented in Table 2. 


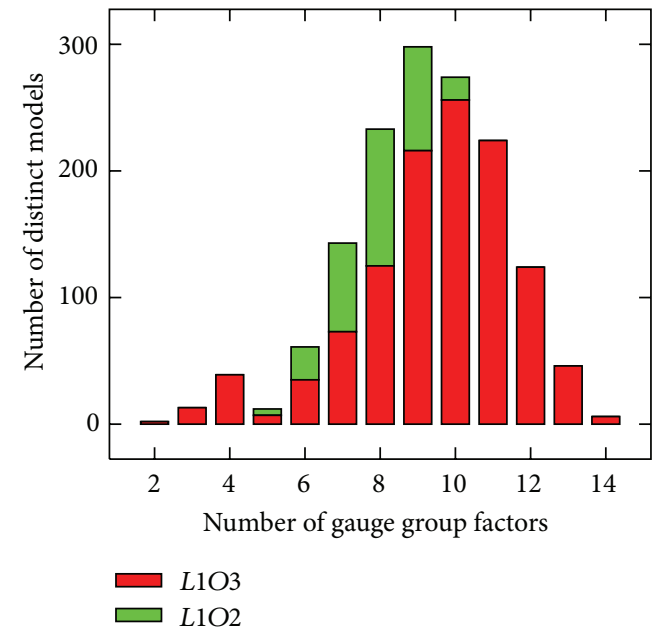

(a) Number of gauge group factors

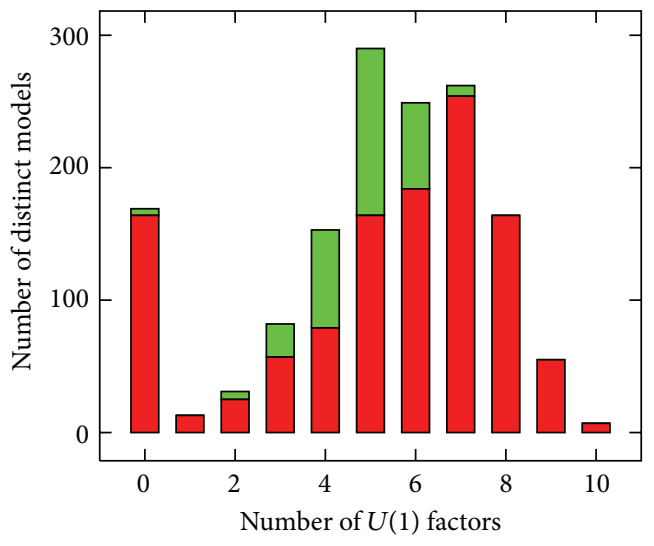

L1O3

$\square \mathrm{L} 1 \mathrm{O} 2$

(c) Number of $U(1)$ factors

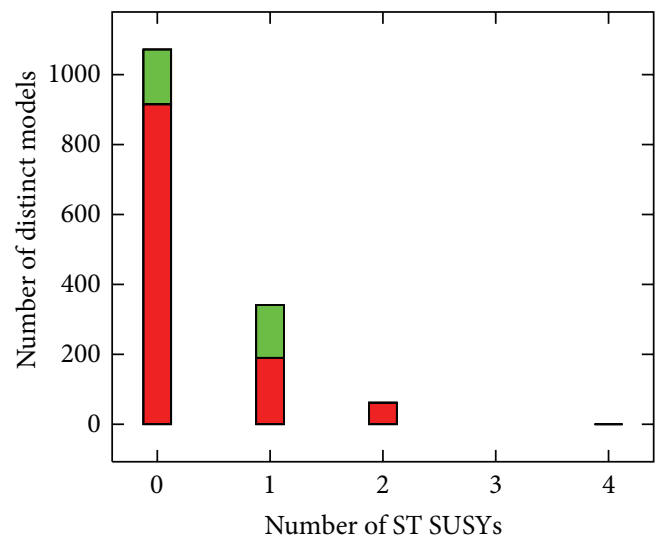

ए1O3

$\square$ L1O2

(b) Number of spacetime SUSYs

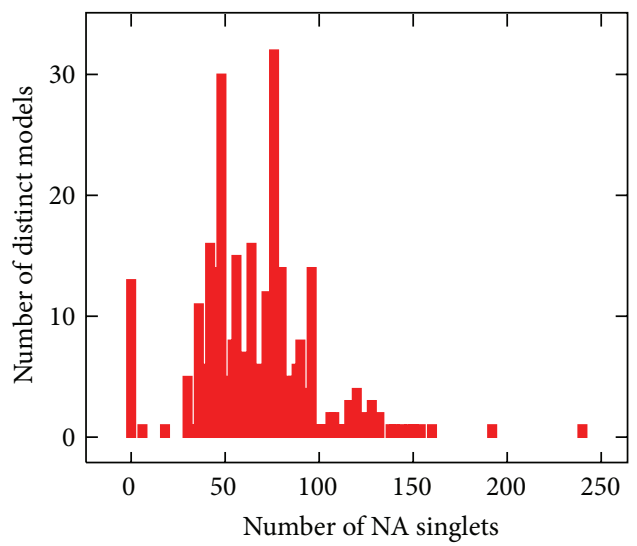

(d) Number of non-Abelian singlets- $\mathrm{L} 1 \mathrm{O} 2$ extension

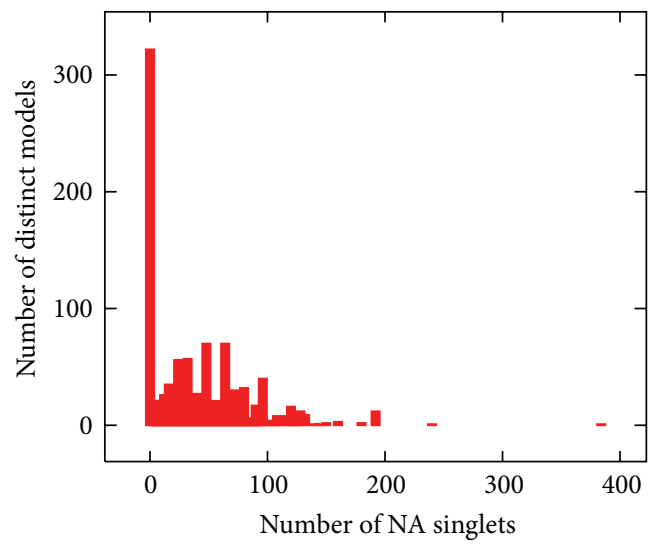

(e) Number of non-Abelian singlets- $\mathrm{L} 1 \mathrm{O} 3$ extension

FIGURE 1: Statistics for the full NAHE variation extension data set. 


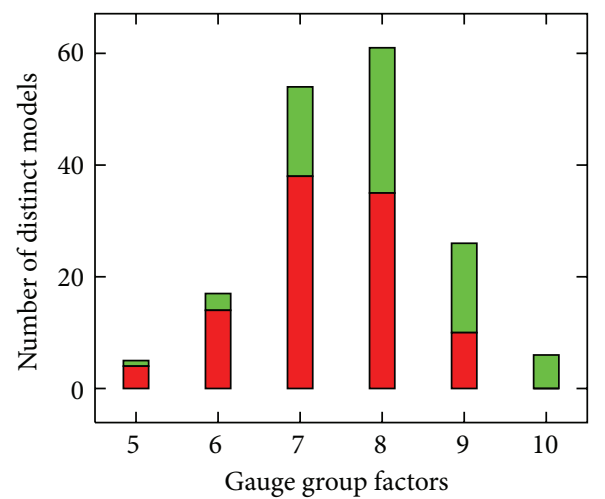

(a) $E_{6}$ gauge group factors

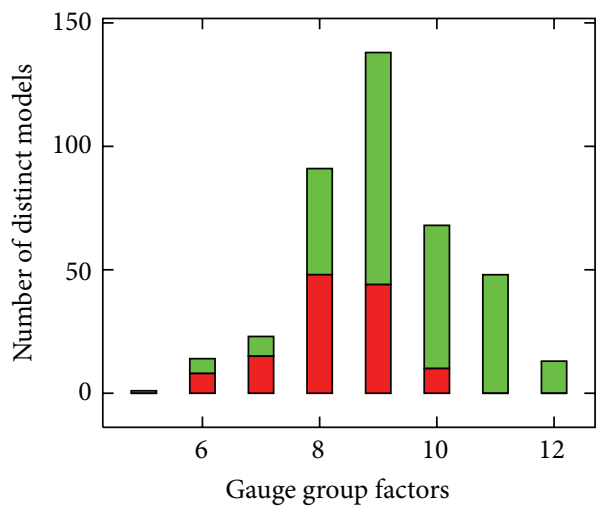

$\square 1 \mathrm{O} 2$

$\square \mathrm{L1O3}$

(c) $\mathrm{SO}(10)$ gauge group factors

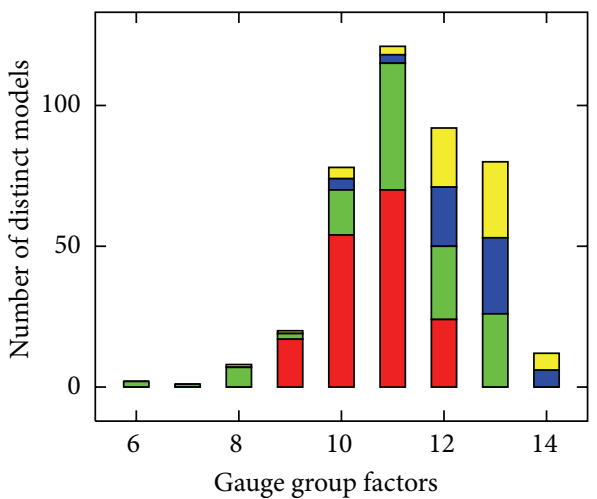

ए $S U(5) \otimes U(1) \quad$ LRS

$\square$ Pati-Salam $\square$ MSSM

(e) Remaining GUTs-gauge group factors

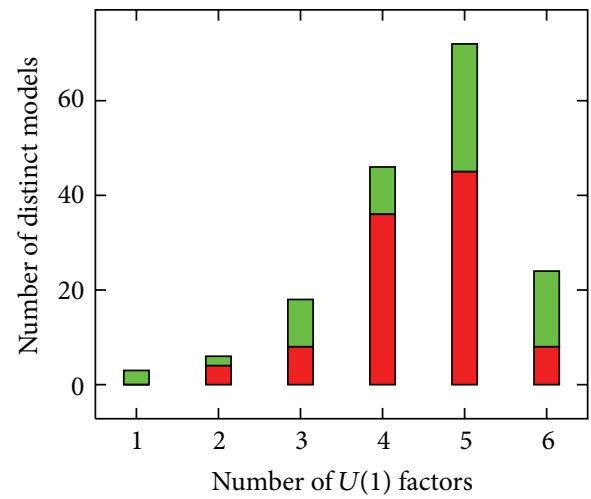

(b) $E_{6}-U(1)$ factors

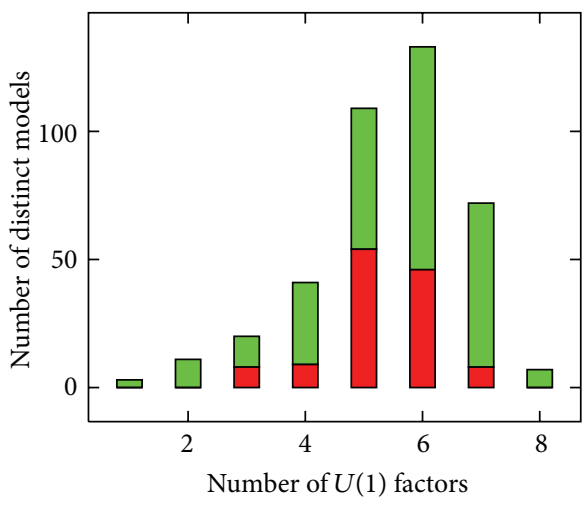

$\mathrm{L} 1 \mathrm{O} 2$

$\square$ L1O3

(d) $S O(10)-U(1)$ factors

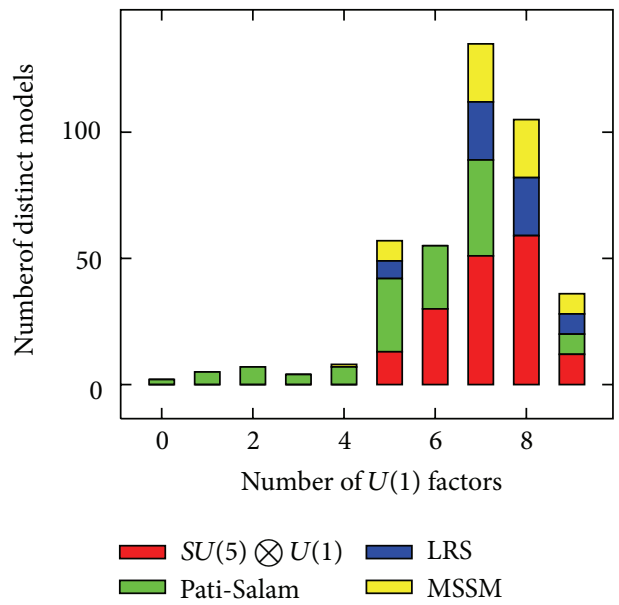

(f) Remaining GUTs-U(1) factors

FIGURE 2: Gauge and $U(1)$ statistics for various GUT models in the NAHE variation extensions data set. 


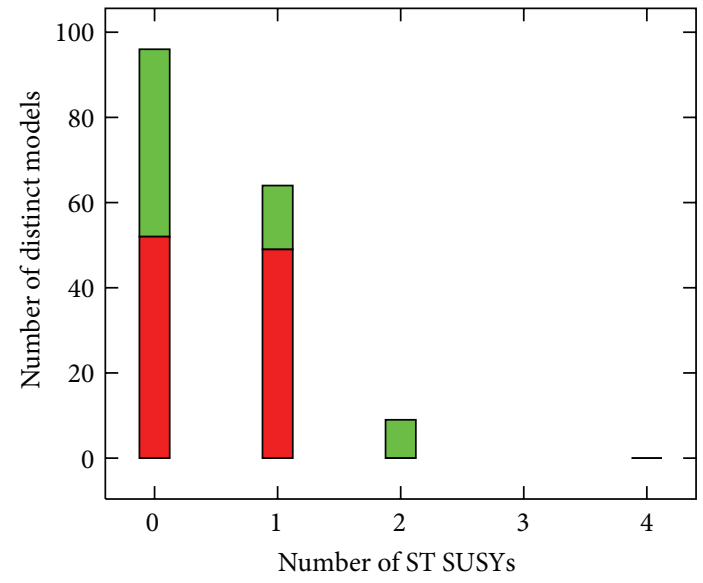

L1O2 $\mathrm{L} 1 \mathrm{O} 3$

(a) $E_{6}$-number of ST SUSYs

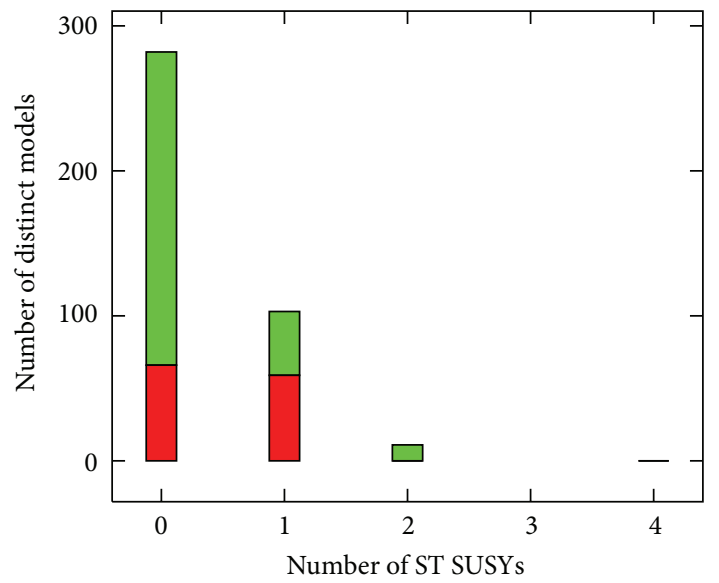

L1O2 $L 1 O 3$

(b) SO(10)-number of ST SUSYs

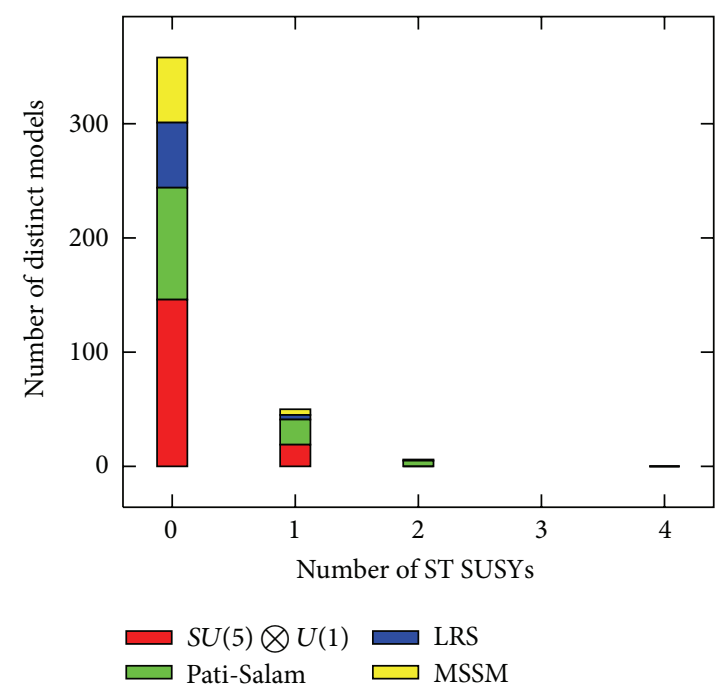

(c) Number of ST SUSYs

FIGURE 3: ST SUSY statistics for various GUT models. Note that only the $E_{6}$ and $S O(10)$ occur from $L 1 O 2$ extensions.

TABLE 1: The basis vectors and GSO coefficients of the NAHE variation arranged into sets of matching boundary conditions. The worldsheet fermions $\psi, x^{i}, \bar{\psi}^{i}, \bar{\eta}^{i}$, and $\bar{\phi}^{i}$ are expressed in a complex basis, while $y^{i}, w^{i}, \bar{y}^{i}$, and $\bar{w}^{i}$ are expressed in a real basis.

\begin{tabular}{|c|c|c|c|c|c|c|c|c|c|c|c|c|c|c|}
\hline Sec & $O$ & $\psi$ & $x^{12}$ & $x^{34}$ & $x^{56}$ & $\bar{\psi}^{1, \ldots, 5}$ & $\bar{\eta}^{1}$ & $\bar{\eta}^{2}$ & $\bar{\eta}^{3}$ & $\bar{\phi}^{1, \ldots, 8}$ & $y^{12} \| \bar{y}^{12}$ & $y^{34} \| \bar{y}^{34}$ & $y^{56} \| \bar{y}^{56}$ & $w^{1, \ldots, 6} \| \bar{w}^{1, \ldots, 6}$ \\
\hline $\overrightarrow{\mathbb{1}}$ & 2 & 1 & 1 & 1 & 1 & $1, \ldots, 1$ & 1 & 1 & 1 & $1, \ldots, 1$ & $1 \| 1$ & $1 \| 1$ & $1 \| 1$ & $1, \ldots, 1 \| 1, \ldots, 1$ \\
\hline$\vec{S}$ & 2 & 1 & 1 & 1 & 1 & $0, \ldots, 0$ & 0 & 0 & 0 & $0, \ldots, 0$ & $0 \| 0$ & $0 \| 0$ & $0 \| 0$ & $0, \ldots, 0 \| 0, \ldots, 0$ \\
\hline$\vec{b}_{1}$ & 2 & 1 & 1 & 0 & 0 & $1, \ldots, 1$ & 1 & 0 & 0 & $0, \ldots, 0$ & $0 \| 0$ & 1|| 1 & $1 \| 1$ & $0, \ldots, 0 \| 0, \ldots, 0$ \\
\hline$\vec{b}_{2}$ & 2 & 1 & 0 & 1 & 0 & $1, \ldots, 1$ & 0 & 1 & 0 & $0, \ldots, 0$ & $1 \| 1$ & $0 \| 0$ & $1 \| 1$ & $0, \ldots, 0 \| 0, \ldots, 0$ \\
\hline$\vec{b}_{3}$ & 2 & 1 & 0 & 0 & 1 & $1, \ldots, 1$ & 0 & 0 & 1 & $0, \ldots, 0$ & 1|| 1 & $1 \| 1$ & $0 \| 0$ & $0, \ldots, 0 \| 0, \ldots, 0$ \\
\hline & & & & & & & $k_{i j}=$ & $\begin{array}{c}\overrightarrow{1} \\
\vec{S} \\
\vec{b}_{1} \\
\vec{b}_{2} \\
\vec{b}_{3}\end{array}$ & $\begin{array}{ll}1 & S \\
1 & 0 \\
0 & 0 \\
1 & 1 \\
1 & 1 \\
1 & 1\end{array}$ & $\begin{array}{ccc}\vec{b}_{1} & \vec{b}_{2} & \vec{b}_{3} \\
1 & 1 & 1 \\
0 & 0 & 0 \\
1 & 1 & 1 \\
1 & 1 & 1 \\
1 & 1 & 1\end{array}$ & & & & \\
\hline
\end{tabular}


TABle 2: The particle content for the NAHE variation model. The model also has five $U(1)$ groups and $N=1$ ST SUSY.

\begin{tabular}{lcc}
\hline QTY & $S O(22)$ & $E_{6}$ \\
\hline 30 & 22 & 1 \\
15 & 1 & 27 \\
90 & 1 & 1 \\
15 & 1 & $\frac{27}{27}$ \\
\hline
\end{tabular}

TABLE 3: A summary of the GUT group study with regard to the number of chiral fermion generations in the NAHE variation investigation.

\begin{tabular}{lcc}
\hline GUT & Net chiral generations & Three generations \\
\hline$L 1 O 2 E_{6}$ & Yes & No \\
$L 1 O 2 S O(10)$ & Yes & No \\
$L 1 O 3 E_{6}$ & No & No \\
$L 1 O 3 S O(10)$ & No & No \\
$L 1 O 3 S U(5) \otimes U(1)$ & No & No \\
$L 1 O 3$ Pati-Salam & No & No \\
$L 1 O 3$ L-R symmetric & No & No \\
$L 1 O 3$ MSSM & No & No \\
\hline
\end{tabular}

The observable sector is generally regarded as being the $E_{6}$; however, contributions to the observable sector may come from the breaking of the $S O(22)$. As compared to the NAHE set, the large number of $U(1)$ s and non-Abelian singlets is less phenomenologically favorable; however, the quantities of both can be reduced drastically which are shown in the statistics for single-layer extensions.

In Section 2, layer 1, order 2 ( $L 1 O 2)$ extensions of the NAHE variation are investigated, with a focus on statistics. In Section 3, L1O3 extensions are similarly examined. In Section 4, the statistics of GUT and of spacetime supersymmetries of both orders are determined. Section 5 offers an example of a near mirrored model, and Section 6 reviews the findings of the prior sections.

\section{Layer 1, Order 2 Extensions}

There were 309 quasi-unique models out of 1, 315,328 total consistent models built given the input parameters. A redundancy related to the rotation of the gauge groups, discussed in detail in [58], is also present. Duplicate models within the set of 309 were removed by hand. Approximately $2 \%$ of the models in the data set without rank cuts were duplicates, while none of the models with rank cuts had duplicates. The gauge group content of those models is presented in Table 5(a).

The most common gauge group in this data set is $U(1)$, while the most common non-Abelian gauge group is $S U(2)$, though less than half of the models contain it. The other pertinent feature of these models is the presence of nonsimply laced gauge groups with high rank. The $S O(2 n+1)$ groups range from rank 2 up to rank 10. Finally, about one third of the models retain their $E_{6}$ symmetry. The stability of the $E_{6}$ is in contrast to the more common breaking of $S O(10)$, the observable sector, in NAHE-based models [58]. These models will be revisited later with the $E_{6}$ treated as an

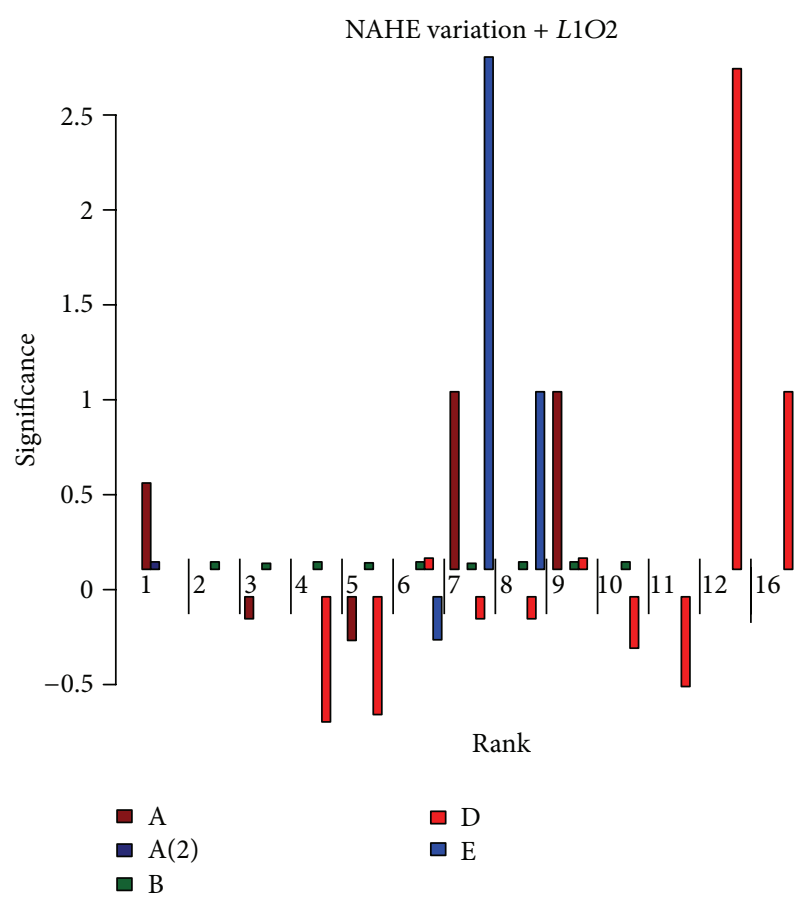

FIgURE 4: The significance values for models in the NAHE variation L1O2 extensions with regard to ST SUSY. Any (absolute) significance values greater than three indicate a strong statistical significance.

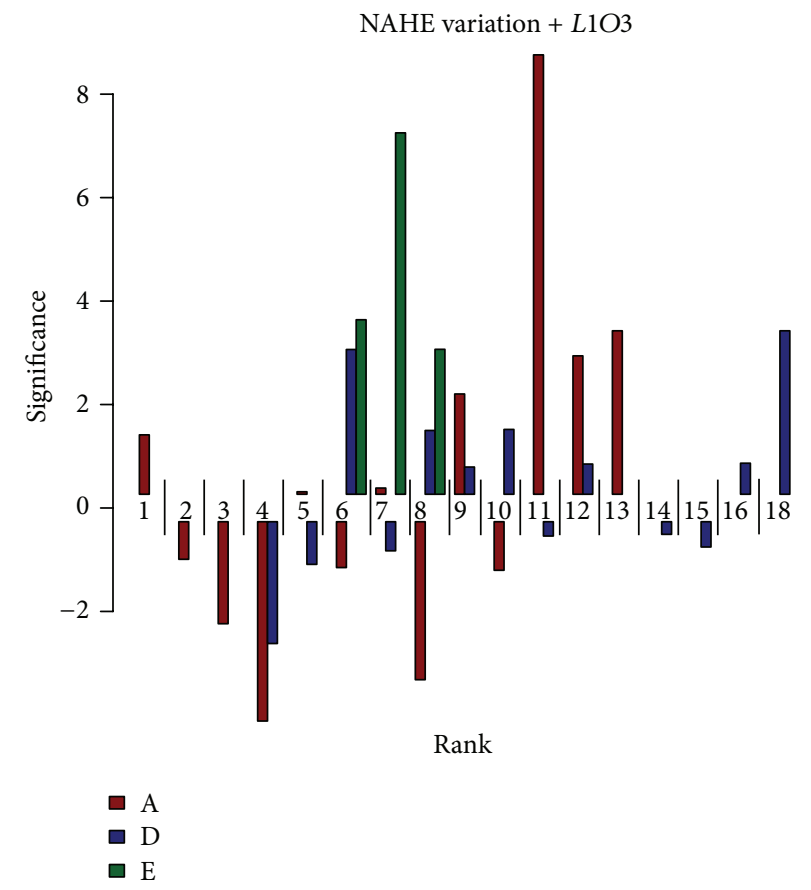

FIGURE 5: The significance values for models in the NAHE variation L1O3 extensions. Any (absolute) significance values greater than three indicate a strong statistical significance. 


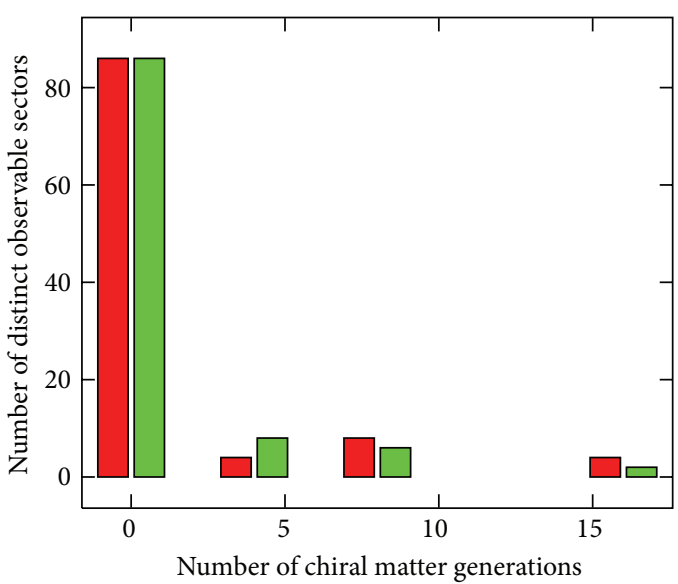

प With duplicates

Qu Without duplicates

(a) $E_{6}$-chiral matter generations

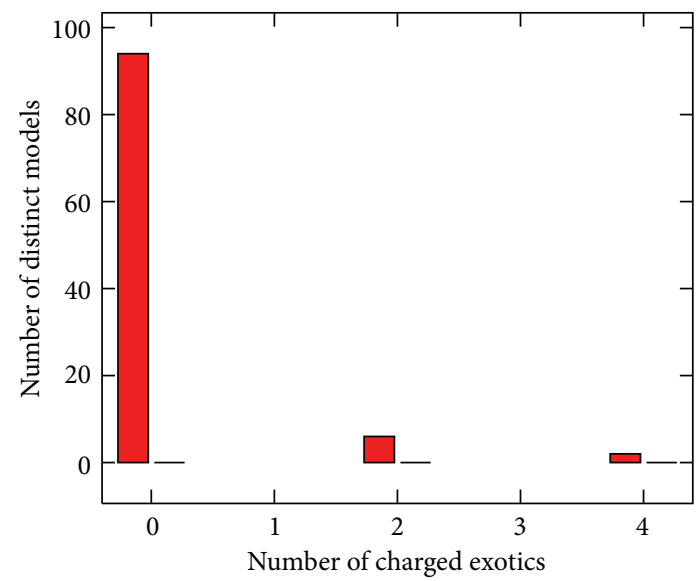

Пए $L 1 O 2$

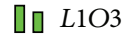

(c) $E_{6}$-charged exotics

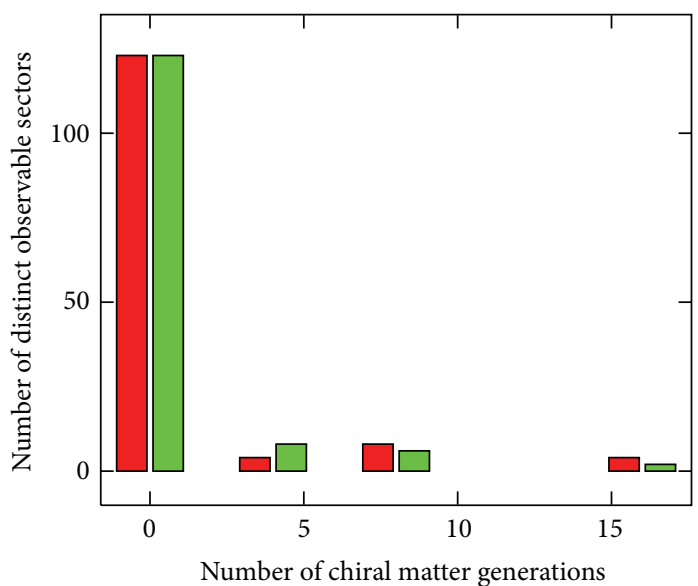

Wu With duplicates

[u Without duplicates

(b) $S O(10)$-chiral matter generations

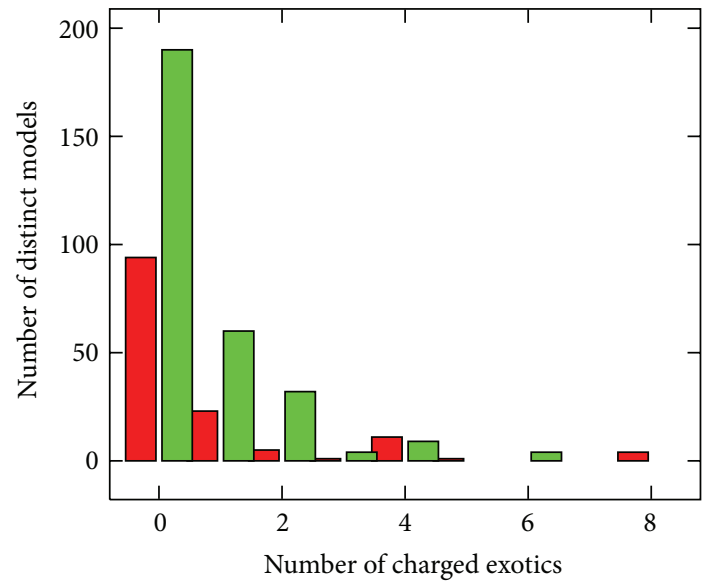

पㄴ $L 1 O 2$ ㅁ $L 1 O 3$

(d) $S O(10)$-charged exotics

FIGURE 6: The number of chiral matter generations and charged exotics for $E_{6}$ and $S O(10)$ models in the NAHE variation extensions.

observable sector gauge group, and the number of chiral matter generations they have will be statistically examined.

Also of interest regarding the gauge group content of this data set is the number of gauge group factors present in each model; see Figure 1(a). The distribution of the number of gauge group factors across the unique models peaks around 8 , suggesting that, roughly, the most common effect of $L 1 O 2$ extension is the breaking of only one group factor. In a few models, some of the factors have enhancements, typically the $U(1)$ groups. Additional adjoint content distributions are provided in Figure 1(c), with GUT model distributions presented in Table 4, but will not be discussed in detail here.

Regarding the matter content, the number of ST SUSYs is plotted in Figure 1(b), and the number of non-Abelian singlets is plotted in Figure 1(d). It is clear from the latter that the number of non-Abelian singlets can get quite high. While most models have between 50 and 80 , there can be up to 250 non-Abelian singlets in a model. This implies that many models in this data set cannot be viable candidates for quasirealistic or realistic models.

\section{Layer 1, Order 3 Extensions}

As was the case with the NAHE extensions, there are more distinct NAHE variation $L 1 O 3$ extensions than $L 1 O 2$ extensions. Out of 442, 272 models built 1, 166 of them were unique. Based on the order- 2 redundancies, the systematic uncertainty for this data set is estimated to be $2 \%$. Their gauge group content is tabulated in Table 5(b). 
TABLE 4: The GUT group content of the NAHE variation extensions data set.

\begin{tabular}{|c|c|c|c|c|}
\hline \multirow[b]{2}{*}{ GUT group } & \multicolumn{2}{|c|}{ L1O2 } & \multicolumn{2}{|c|}{$L 1 O 3$} \\
\hline & $\begin{array}{c}\text { Number of unique } \\
\text { models }\end{array}$ & $\begin{array}{l}\text { Percentage of unique } \\
\text { models }\end{array}$ & $\begin{array}{l}\text { Number of unique } \\
\text { models }\end{array}$ & $\begin{array}{l}\text { Percentage of unique } \\
\text { models }\end{array}$ \\
\hline$E_{6}$ & 101 & $32.69 \%$ & 68 & $5.832 \%$ \\
\hline$S O(10)$ & 125 & $40.45 \%$ & 271 & $23.24 \%$ \\
\hline$S U(5) \otimes U(1)$ & 0 & $0 \%$ & 165 & $14.15 \%$ \\
\hline$S U(4) \otimes S U(2) \otimes S U(2)$ & 0 & $0 \%$ & 125 & $10.72 \%$ \\
\hline$S U(3) \otimes S U(2) \otimes S U(2)$ & 0 & $0 \%$ & 61 & $5.232 \%$ \\
\hline$S U(3) \otimes S U(2) \otimes U(1)$ & 0 & $0 \%$ & 63 & $5.403 \%$ \\
\hline
\end{tabular}

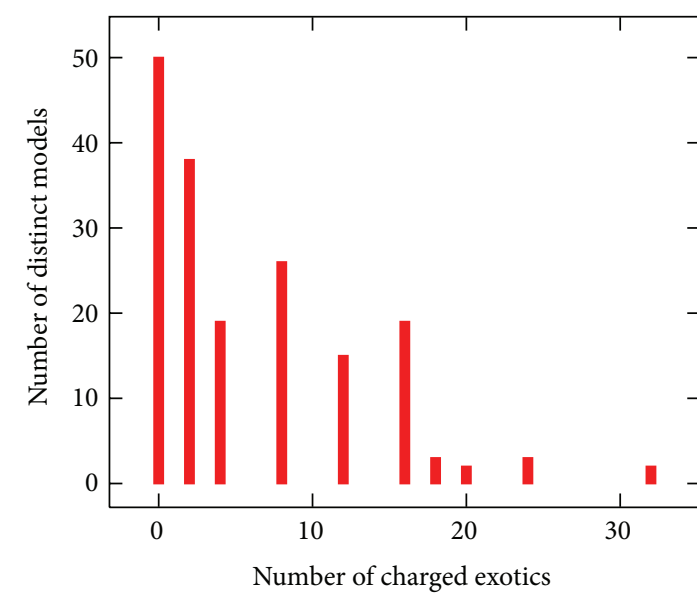

(a) $S U(5) \otimes U(1)$

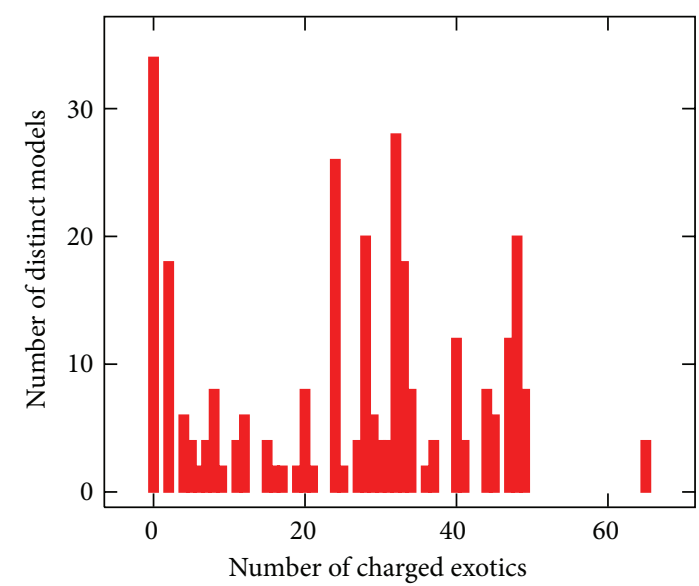

(c) Left-Right Symmetric

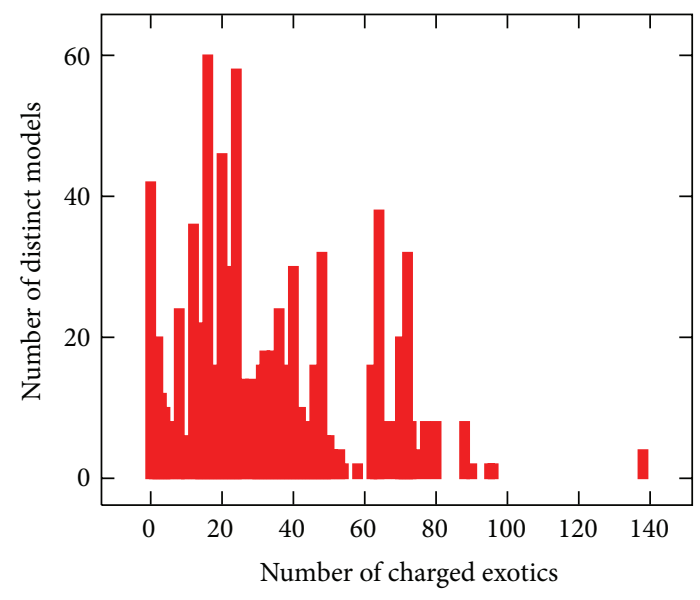

(b) Pati-Salam

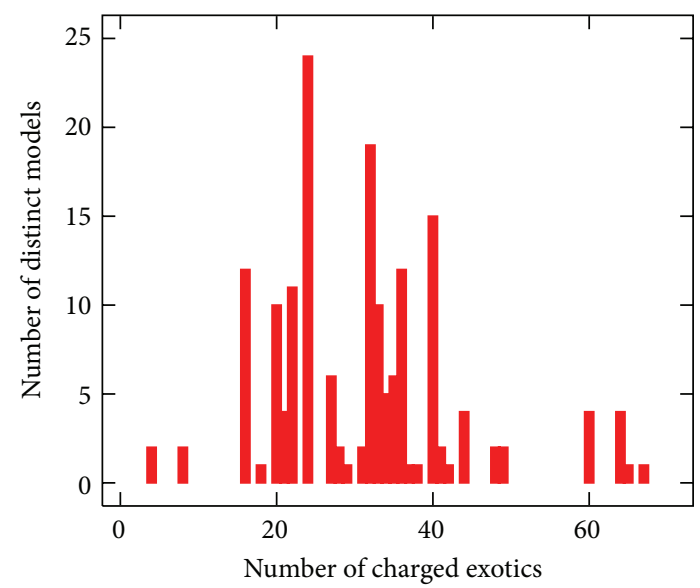

(d) MSSM

FIGURE 7: The number of charged exotics for $S U(5) \otimes U(1)$, Pati-Salam, Left-Right Symmetric, and MSSM-like models in the NAHE variation extensions.

As was the case with the $L 1 O 2$ data set, $U(1)$ is the most common gauge group. However, the percentage is significantly lower here, about $86 \%$ as opposed to $98 \%$. This suggests that some of the added basis vectors are unifying the five $U(1) s$ in the NAHE variation into larger gauge groups. Also of note is the number of models with gauge groups of rank higher than 11 . In the $\mathrm{L} 1 \mathrm{O} 2$ data set, there were only three models of this type, about $1 \%$. In the $L 1 O 3$ data set, there were 28 models with this property, about $2.4 \%$.
While it may seem from Table 5(b) that the order-3 models are more prone to enhancements, Figure 1(a) makes it clear that is not the case. The distribution of the number of gauge group factors for a model peaks between 9 and 11 factors, as opposed to the peak at 8 factors for the order2 models. However, there are several models with enhancements, even some models with as few as 2 distinct gauge group factors in them, something not seen with the order2 models. This implies there is a class of order-3 basis vectors 


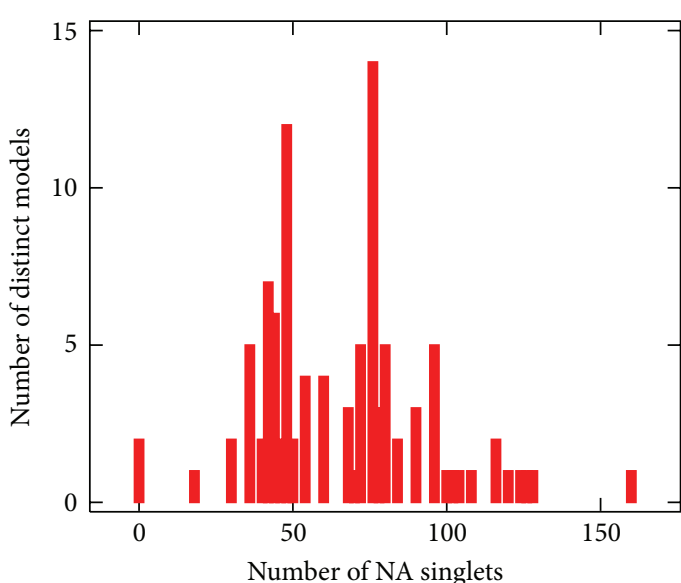

(a) $E_{6}$-number of NA singlets ( $\mathrm{L1O2}$ )

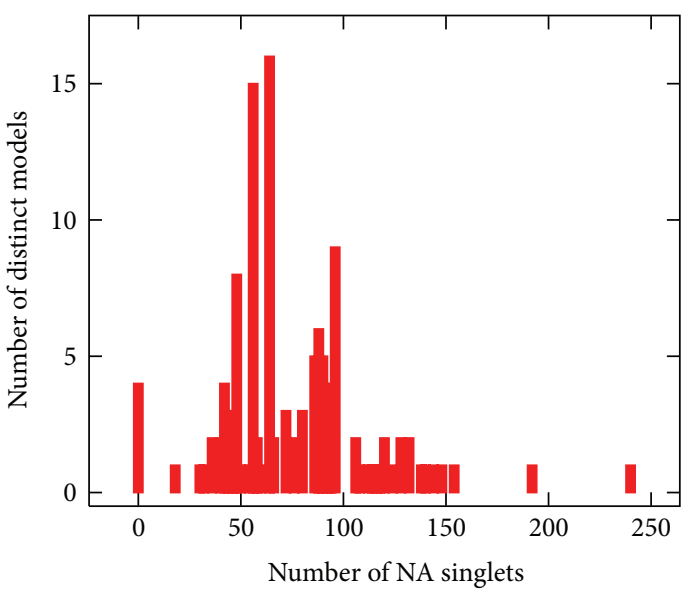

(c) $\mathrm{SO}(10)$-number of NA singlets $(\mathrm{L1O2})$

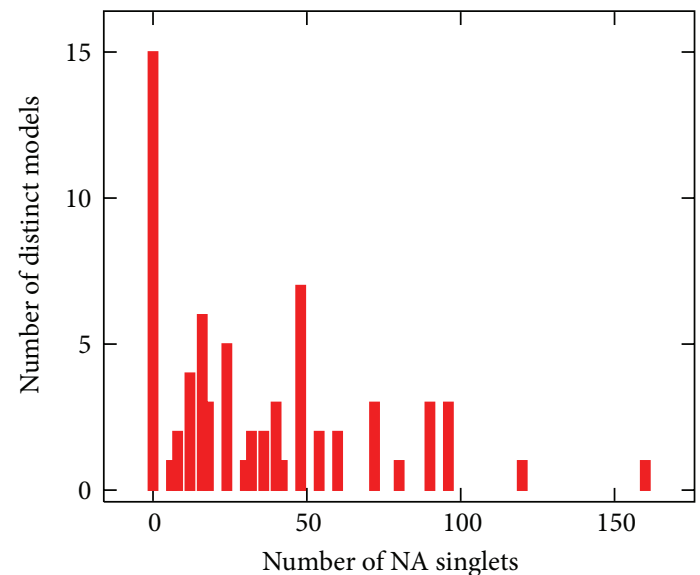

(b) $E_{6}$-number of NA singlets (L1O3)

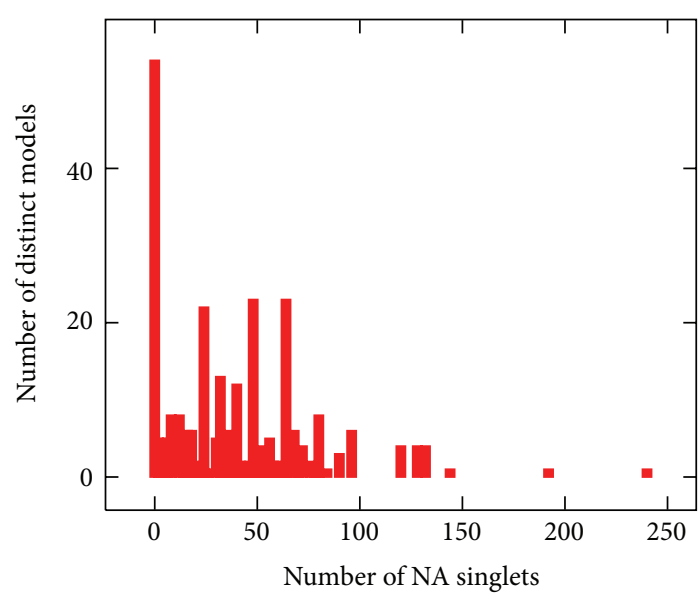

(d) $\mathrm{SO}(10)$-number of NA singlets ( $\mathrm{L1O3}$ )

FIGURE 8: NA singlet statistics for the $E_{6}$ and $S O(10)$ models in the NAHE variation extenstions data set.

that greatly enhances the gauge group symmetries, while most order-3 models break them.

The number of $U(1)$ gauge groups per model is plotted in Figure 1(c). The distribution of $U(1)$ peaks between 5 and 7. More interestingly, a nontrivial number of models do not have $U(1)$ symmetries at all. This implies, when combined with Figure 1(a), that in some models the $U(1)$ s are enhancing larger (but still small relative to $S O(22)$ and $E_{6}$ ) gauge groups. The mechanism producing this effect warrants further study, as it could be used to reduce the number of $U(1)$ factors for order-layer combinations that tend to produce too many $U(1)$ s. The frequency of the GUT groups is presented in Table 4.

The number of ST SUSYs is presented in Figure 1(b). While there are a statistically significant number of enhanced ST SUSYs (expected from models with odd-ordered right movers), the majority of these models has $N=0$ ST SUSY.

The number of non-Abelian singlets is plotted in Figure 1(e). The distribution of non-Abelian singlets indicates that a large number of models do not have any non-Abelian singlets. It is possible that this is related to the number of models with no $U(1)$ factors.

\section{Models with GUT Groups}

As a parallel to the NAHE extension study, the subsets of models containing the GUT groups $E_{6}, S O(10), S U(5) \otimes U(1)$, $S U(4) \otimes S U(2) \otimes S U(2)$ (Pati-Salam), $S U(3) \otimes S U(2) \otimes S U(2)$ (Left-Right Symmetric), and $S U(3) \otimes S U(2) \otimes U(1)(M S S M)$ are examined (see Figure 2). Like the NAHE study, the usual statistics will be reported along with the number of net chiral generations for models containing the GUT groups in question. If there is more than one way to configure an observable sector, each configuration will be counted when tallying the charged exotics and net chiral generations. For example, a model may have two $E_{6}$ groups with different matter representations. Each one would be counted individually when examining the number of charged exotics and net chiral generations. 


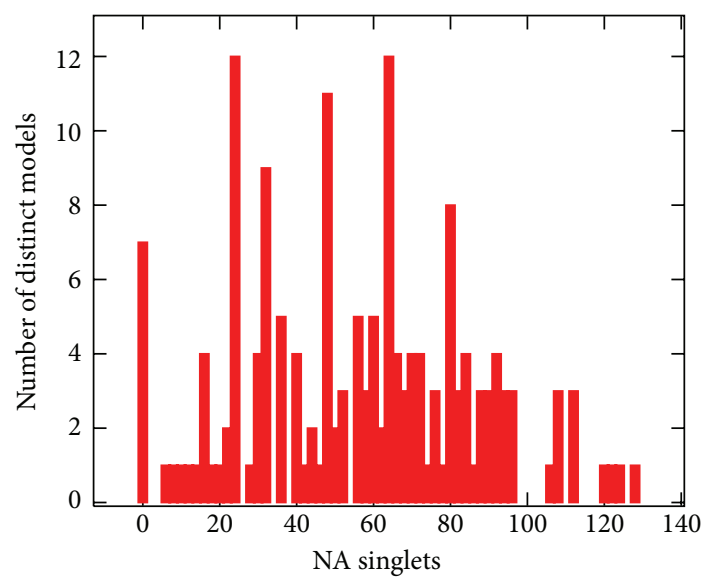

(a) $S U(5) \otimes U(1)$

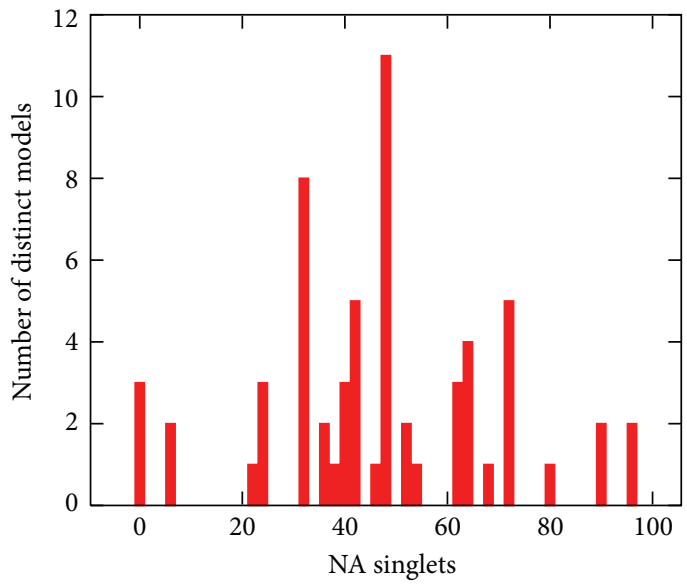

(c) Left-Right Symmetric

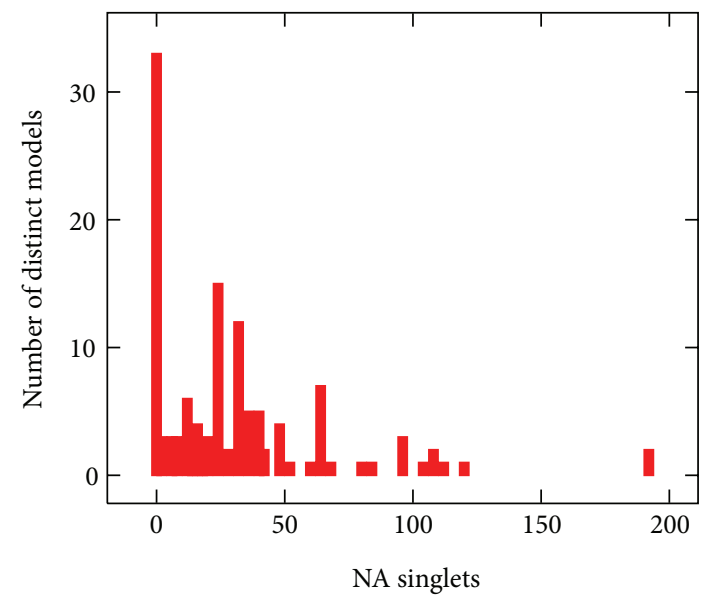

(b) Pati-Salam

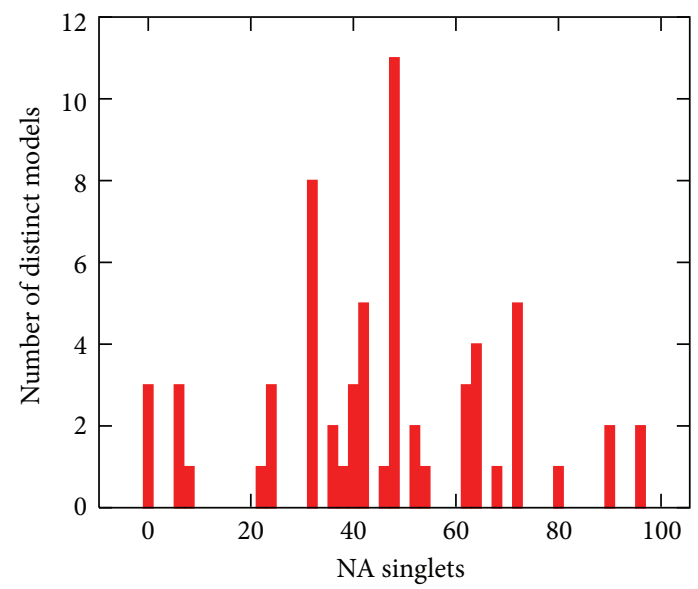

(d) MSSM

FIGURE 9: NA singlet statistics for the $S U(5) \otimes U(1)$, Pati-Salam, Left-Right Symmetric, and MSSM-like models. Note that these only arise as L1O3 extensions.

In order to calculate the net number of chiral fermion generations, we utilize the following expressions:

$$
\begin{array}{ll}
E_{6} & \left|N_{27}-N_{\overline{27}}\right| \\
S O(10) & \left|N_{16}-N_{\overline{16}}\right| \\
S U(5) \otimes U(1) & \left|\min \left(N_{10}, N_{\overline{5}}\right)-\min \left(N_{\overline{10}}, N_{5}\right)\right| \\
\text { Pati-Salam } & \left|N_{(4,2,1)}-N_{(\overline{4}, 2,1)}\right| \\
\text { Left-Right Symmetric } & \left|N_{(3,2,1)}-N_{(\overline{3}, 2,1)}\right| \\
\text { MSSM } & \left|N_{(3,2)}-N_{(\overline{3}, 2)}\right|,\left|N_{(3,1)}-N_{(\overline{3}, 1)}\right| .
\end{array}
$$

Upon analysis, it is found that the $\mathrm{L} 1 \mathrm{O} 2$ extensions yield $E_{6}$ and $S O(10)$ observable sectors with net chiral generations while no models with $S U(5) \otimes U(1), S O(6) \otimes S O(4), S U(3) \otimes$ $S U(2) \otimes S U(2)$, nor $S U(3) \otimes S U(2) \otimes U(1)$ have this property. This is a consequence of the fact that the latter groups only arise from $\mathrm{L}_{103}$ extensions which are not conducive to production of net chiral generations. The distribution of net chiral generations, as well as charged exotic matter, by gauge group is provided in Figures 6 and 7. The distributions of number of non-Abelian singlets, by gauge group, can be found in Figures 8 and 9.
In addition to matter content, the hidden sector gauge content is tabulated for each of the aforementioned gauge groups: Tables 6, 7, and 8. We can see from Table 4 that the NAHE variation extensions favor $E_{6}$ and $S O(10)$ over the other groups. This is easily understood as $E_{6}$ is already present and the breaking $E_{6}$ to $S O(10)$ is rather straight forward. However, in order to produce the low-rank $S U(n+1)$ groups, either the $U(1) s$ must be enhanced or there must be significant breaking of either the $E_{6}$ or $S O(22)$. However, neither of these readily occur with a single layer or at low order.

4.1. ST SUSYs. The distributions of ST SUSYs for the entire data set can be found in Figure 1(b) with a breakdown by gauge group in Figure 3.

The $\mathrm{L} 1 \mathrm{O} 2$ models all have the same distributions regardless of which GUT is chosen. In these models, the gauge content does not statistically couple with the ST SUSY. For the L1O3 models, however, some of the GUT groups do appear to have such a coupling. In particular, the occurrence of $E_{6}$ 
TABLE 5: The gauge group content of the NAHE variation data set.

(a) Layer 1, order 2

\begin{tabular}{|c|c|c|}
\hline Gauge group & $\begin{array}{c}\text { Number of unique } \\
\text { models }\end{array}$ & $\begin{array}{l}\text { Percentage of unique } \\
\text { models }\end{array}$ \\
\hline$S U(2)$ & 131 & $42.39 \%$ \\
\hline$S U(2)^{(2)}$ & 18 & $5.825 \%$ \\
\hline$S U(4)$ & 33 & $10.68 \%$ \\
\hline$S U(6)$ & 99 & $32.04 \%$ \\
\hline$S U(8)$ & 1 & $0.3236 \%$ \\
\hline$S U(10)$ & 1 & $0.3236 \%$ \\
\hline$S O(5)$ & 18 & $5.825 \%$ \\
\hline $\mathrm{SO}(7)$ & 12 & $3.883 \%$ \\
\hline $\mathrm{SO}(9)$ & 18 & $5.825 \%$ \\
\hline$S O(11)$ & 14 & $4.531 \%$ \\
\hline$S O(13)$ & 18 & $5.825 \%$ \\
\hline$S O(15)$ & 12 & $3.883 \%$ \\
\hline$S O(17)$ & 18 & $5.825 \%$ \\
\hline$S O(19)$ & 18 & $5.825 \%$ \\
\hline$S O(21)$ & 18 & $5.825 \%$ \\
\hline$S O(8)$ & 30 & $9.709 \%$ \\
\hline$S O(10)$ & 125 & $40.45 \%$ \\
\hline$S O(12)$ & 38 & $12.3 \%$ \\
\hline$S O(14)$ & 33 & $10.68 \%$ \\
\hline$S O(16)$ & 33 & $10.68 \%$ \\
\hline$S O(18)$ & 38 & $12.3 \%$ \\
\hline$S O(20)$ & 36 & $11.65 \%$ \\
\hline$S O(22)$ & 31 & $10.03 \%$ \\
\hline$S O(24)$ & 2 & $0.6472 \%$ \\
\hline $\mathrm{SO}(32)$ & 1 & $0.3236 \%$ \\
\hline$E_{6}$ & 101 & $32.69 \%$ \\
\hline$E_{7}$ & 3 & $0.9709 \%$ \\
\hline$E_{8}$ & 1 & $0.3236 \%$ \\
\hline$U(1)$ & 304 & $98.38 \%$ \\
\hline
\end{tabular}

(b) Layer 1, order 3

\begin{tabular}{lcc}
\hline Gauge group & $\begin{array}{c}\text { Number of unique } \\
\text { models }\end{array}$ & $\begin{array}{c}\text { Percentage of unique } \\
\text { models }\end{array}$ \\
\hline$S U(2)$ & 731 & $62.69 \%$ \\
$S U(3)$ & 128 & $10.98 \%$ \\
$S U(4)$ & 355 & $30.45 \%$ \\
$S U(5)$ & 165 & $14.15 \%$ \\
$S U(6)$ & 167 & $14.32 \%$ \\
$S U(7)$ & 75 & $6.432 \%$ \\
$S U(8)$ & 143 & $12.26 \%$ \\
$S U(9)$ & 164 & $14.07 \%$ \\
$S U(10)$ & 169 & $14.49 \%$ \\
$S U(11)$ & 137 & $11.75 \%$ \\
$S U(12)$ & 56 & $4.803 \%$ \\
$S U(13)$ & 4 & $0.3431 \%$ \\
\hline
\end{tabular}

(b) Continued.

\begin{tabular}{lcc}
\hline Gauge group & $\begin{array}{c}\text { Number of unique } \\
\text { models }\end{array}$ & $\begin{array}{c}\text { Percentage of unique } \\
\text { models }\end{array}$ \\
\hline$S U(14)$ & 1 & $0.08576 \%$ \\
$S O(8)$ & 376 & $32.25 \%$ \\
$S O(10)$ & 271 & $23.24 \%$ \\
$S O(12)$ & 151 & $12.95 \%$ \\
$S O(14)$ & 81 & $6.947 \%$ \\
$S O(16)$ & 106 & $9.091 \%$ \\
$S O(18)$ & 28 & $2.401 \%$ \\
$S O(20)$ & 69 & $5.918 \%$ \\
$S O(22)$ & 5 & $0.4288 \%$ \\
$S O(24)$ & 11 & $0.9434 \%$ \\
$S O(28)$ & 13 & $1.115 \%$ \\
$S O(30)$ & 1 & $0.08576 \%$ \\
$S O(32)$ & 2 & $0.1715 \%$ \\
$S O(36)$ & 1 & $0.08576 \%$ \\
$E_{6}$ & 68 & $5.832 \%$ \\
$E_{7}$ & 24 & $2.058 \%$ \\
$E_{8}$ & 9 & $0.7719 \%$ \\
$U(1)$ & 1002 & $85.93 \%$ \\
\hline & &
\end{tabular}

models $N=2$ ST SUSY is disproportionately high while $S U(5) \otimes U(1)$, Left-Right Symmetric, and MSSM models with $N=1$ ST SUSY have a reduced occurrence. As all of the models containing these GUTs have at least a single $U(1)$, there could be a correlation between the number of $U(1) \mathrm{s}$ and the number of ST SUSYs. Further investigations of these findings show several statistical couplings for higher ST SUSY models containing certain gauge group factors. The methodology used to analyze these couplings was detailed in [58]. The observed significances are plotted in Figures 4 and 5 for the L1O2 and L1O3 NAHE variation extensions, respectively.

While there are no significant gauge groups in the $\mathrm{L} 1 \mathrm{O} 2$ extensions, several groups are significant with regard to enhanced ST SUSYs in the NAHE L1O3 extensions. In particular, the three exceptional groups, as well as $S O(12), S U(12)$, $S U(13), S U(14)$, and $S O(36)$, all have a significant statistical correlation with the average number of ST SUSYs. This is likely due to the additional basis vector adding a gravitino generating sector, which is common with odd-order extensions, and additional roots for the gauge groups. Further analysis will be needed to confirm the cause of this significance. It is also worth noting that one group, $S U(5)$, has a negative impact on ST SUSYs. If this trend occurs for more oddordered extensions of the NAHE variation, it may affect the viability of realistic flipped-SU(5) models derived from this variation.

\section{Models with Mirroring}

The larger sets of matching boundary conditions, seen in Table 1, are expected to lead to models with mirrored gauge 
TABLE 6: The hidden sector gauge group content for the NAHE variation extension models with $E_{6}$ observable.

(a) Layer 1, order 2

\begin{tabular}{lcc}
\hline Gauge group & $\begin{array}{c}\text { Number of unique } \\
\text { models }\end{array}$ & $\begin{array}{c}\text { Percentage of unique } \\
\text { models }\end{array}$ \\
\hline$S U(2)$ & 14 & $13.86 \%$ \\
$S U(2)^{(2)}$ & 8 & $7.921 \%$ \\
$S U(4)$ & 10 & $9.901 \%$ \\
$S O(5)$ & 6 & $5.941 \%$ \\
$S O(7)$ & 2 & $1.98 \%$ \\
$S O(9)$ & 6 & $5.941 \%$ \\
$S O(11)$ & 6 & $5.941 \%$ \\
$S O(13)$ & 6 & $5.941 \%$ \\
$S O(15)$ & 2 & $1.98 \%$ \\
$S O(17)$ & 6 & $5.941 \%$ \\
$S O(19)$ & 8 & $7.921 \%$ \\
$S O(21)$ & 6 & $5.941 \%$ \\
$S O(8)$ & 8 & $7.921 \%$ \\
$S O(10)$ & 14 & $13.86 \%$ \\
$S O(12)$ & 14 & $13.86 \%$ \\
$S O(14)$ & 9 & $8.911 \%$ \\
$S O(16)$ & 9 & $8.911 \%$ \\
$S O(18)$ & 14 & $13.86 \%$ \\
$S O(20)$ & 12 & $11.88 \%$ \\
$S O(22)$ & 8 & $7.921 \%$ \\
$E_{8}$ & 1 & $0.9901 \%$ \\
$U(1)$ & 101 & $100 \%$ \\
\hline & &
\end{tabular}

(b) Layer 1, order 3

\begin{tabular}{lcc}
\hline Gauge group & $\begin{array}{c}\text { Number of unique } \\
\text { models }\end{array}$ & $\begin{array}{c}\text { Percentage of unique } \\
\text { models }\end{array}$ \\
\hline$S U(2)$ & 31 & $45.59 \%$ \\
$S U(3)$ & 1 & $1.471 \%$ \\
$S U(4)$ & 12 & $17.65 \%$ \\
$S U(6)$ & 4 & $5.882 \%$ \\
$S U(8)$ & 6 & $8.824 \%$ \\
$S U(9)$ & 10 & $14.71 \%$ \\
$S U(10)$ & 8 & $11.76 \%$ \\
$S U(11)$ & 5 & $7.353 \%$ \\
$S U(12)$ & 4 & $5.882 \%$ \\
$S U(13)$ & 1 & $1.471 \%$ \\
$S O(8)$ & 9 & $13.24 \%$ \\
$S O(10)$ & 15 & $22.06 \%$ \\
$S O(12)$ & 12 & $17.65 \%$ \\
$S O(14)$ & 5 & $7.353 \%$ \\
$S O(16)$ & 3 & $4.412 \%$ \\
$S O(18)$ & 4 & $5.882 \%$ \\
$S O(20)$ & 2 & $2.941 \%$ \\
$S O(22)$ & 2 & $2.941 \%$ \\
$E_{8}$ & 2 & $2.941 \%$ \\
$U(1)$ & 68 & $100 \%$ \\
\hline & &
\end{tabular}

TABLE 7: The hidden sector gauge group content for the NAHE variation extension models with $S O(10)$ observable.

(a) Layer 1, order 2

\begin{tabular}{lcc}
\hline Gauge group & $\begin{array}{c}\text { Number of unique } \\
\text { models }\end{array}$ & $\begin{array}{c}\text { Percentage of unique } \\
\text { models }\end{array}$ \\
\hline$S U(2)$ & 23 & $18.4 \%$ \\
$S U(2)^{(2)}$ & 8 & $6.4 \%$ \\
$S U(4)$ & 10 & $8 \%$ \\
$S U(6)$ & 10 & $8 \%$ \\
$S O(5)$ & 6 & $4.8 \%$ \\
$S O(7)$ & 2 & $1.6 \%$ \\
$S O(9)$ & 6 & $4.8 \%$ \\
$S O(11)$ & 6 & $4.8 \%$ \\
$S O(13)$ & 6 & $4.8 \%$ \\
$S O(15)$ & 2 & $1.6 \%$ \\
$S O(17)$ & 6 & $4.8 \%$ \\
$S O(19)$ & 8 & $6.4 \%$ \\
$S O(21)$ & 6 & $4.8 \%$ \\
$S O(8)$ & 8 & $6.4 \%$ \\
$S O(12)$ & 35 & $28 \%$ \\
$S O(14)$ & 10 & $8 \%$ \\
$S O(16)$ & 10 & $8 \%$ \\
$S O(18)$ & 14 & $11.2 \%$ \\
$S O(20)$ & 12 & $9.6 \%$ \\
$S O(22)$ & 9 & $7.2 \%$ \\
$E_{6}$ & 14 & $11.2 \%$ \\
$E_{7}$ & 1 & $0.8 \%$ \\
$U(1)$ & 125 & $100 \%$ \\
\hline & &
\end{tabular}

(b) Layer 1, order 3

\begin{tabular}{lcc}
\hline Gauge group & $\begin{array}{c}\text { Number of unique } \\
\text { models }\end{array}$ & $\begin{array}{c}\text { Percentage of unique } \\
\text { models }\end{array}$ \\
\hline$S U(2)$ & 155 & $57.2 \%$ \\
$S U(3)$ & 27 & $9.963 \%$ \\
$S U(4)$ & 59 & $21.77 \%$ \\
$S U(5)$ & 14 & $5.166 \%$ \\
$S U(6)$ & 59 & $21.77 \%$ \\
$S U(7)$ & 22 & $8.118 \%$ \\
$S U(8)$ & 24 & $8.856 \%$ \\
$S U(9)$ & 36 & $13.28 \%$ \\
$S U(10)$ & 26 & $9.594 \%$ \\
$S U(11)$ & 19 & $7.011 \%$ \\
$S U(12)$ & 11 & $4.059 \%$ \\
$S U(13)$ & 1 & $0.369 \%$ \\
$S U(14)$ & 1 & $0.369 \%$ \\
$S O(8)$ & 48 & $17.71 \%$ \\
$S O(12)$ & 35 & $12.92 \%$ \\
$S O(14)$ & 22 & $8.118 \%$ \\
$S O(16)$ & 10 & $3.69 \%$ \\
$S O(18)$ & 7 & $2.583 \%$ \\
$S O(20)$ & 2 & $0.738 \%$ \\
$S O(22)$ & 3 & $1.107 \%$ \\
$E_{6}$ & 15 & $5.535 \%$ \\
\hline & &
\end{tabular}


(b) Continued.

\begin{tabular}{lcc}
\hline Gauge group & $\begin{array}{c}\text { Number of unique } \\
\text { models }\end{array}$ & $\begin{array}{c}\text { Percentage of unique } \\
\text { models }\end{array}$ \\
\hline$E_{7}$ & 4 & $1.476 \%$ \\
$E_{8}$ & 2 & $0.738 \%$ \\
$U(1)$ & 271 & $100 \%$ \\
\hline
\end{tabular}

TABLE 8: The hidden sector gauge group content for the NAHE variation extension $L 1 O 3$ models with GUT observable.

(a) Left-Right Symmetric

\begin{tabular}{lcc}
\hline Gauge group & $\begin{array}{c}\text { Number of unique } \\
\text { models }\end{array}$ & $\begin{array}{c}\text { Percentage of unique } \\
\text { models }\end{array}$ \\
\hline$S U(4)$ & 12 & $19.67 \%$ \\
$S U(7)$ & 14 & $22.95 \%$ \\
$S U(8)$ & 7 & $11.48 \%$ \\
$S U(9)$ & 9 & $14.75 \%$ \\
$S U(10)$ & 12 & $19.67 \%$ \\
$S U(11)$ & 17 & $27.87 \%$ \\
$S U(12)$ & 2 & $3.279 \%$ \\
$S O(8)$ & 8 & $13.11 \%$ \\
$S O(10)$ & 6 & $9.836 \%$ \\
$U(1)$ & 61 & $100 \%$ \\
\hline
\end{tabular}

(b) MSSM

\begin{tabular}{lcc}
\hline Gauge group & $\begin{array}{c}\text { Number of unique } \\
\text { models }\end{array}$ & $\begin{array}{c}\text { Percentage of unique } \\
\text { models }\end{array}$ \\
\hline$S U(4)$ & 13 & $20.63 \%$ \\
$S U(6)$ & 1 & $1.587 \%$ \\
$S U(7)$ & 14 & $22.22 \%$ \\
$S U(8)$ & 7 & $11.11 \%$ \\
$S U(9)$ & 9 & $14.29 \%$ \\
$S U(10)$ & 12 & $19.05 \%$ \\
$S U(11)$ & 18 & $28.57 \%$ \\
$S U(12)$ & 3 & $4.762 \%$ \\
$S O(8)$ & 8 & $12.7 \%$ \\
$S O(10)$ & 6 & $9.524 \%$ \\
\hline
\end{tabular}

(c) $S U(5) \otimes U(1)$

\begin{tabular}{lcc}
\hline Gauge group & $\begin{array}{c}\text { Number of unique } \\
\text { models }\end{array}$ & $\begin{array}{c}\text { Percentage of unique } \\
\text { models }\end{array}$ \\
\hline$S U(2)$ & 87 & $52.73 \%$ \\
$S U(3)$ & 19 & $11.52 \%$ \\
$S U(4)$ & 28 & $16.97 \%$ \\
$S U(6)$ & 8 & $4.848 \%$ \\
$S U(7)$ & 20 & $12.12 \%$ \\
$S U(8)$ & 23 & $13.94 \%$ \\
$S U(9)$ & 34 & $20.61 \%$ \\
$S U(10)$ & 35 & $21.21 \%$ \\
$S U(11)$ & 32 & $19.39 \%$ \\
$S U(12)$ & 1 & $0.6061 \%$ \\
$S O(8)$ & 22 & $13.33 \%$ \\
$S O(10)$ & 14 & $8.485 \%$ \\
$S O(12)$ & 7 & $4.242 \%$ \\
$S O(14)$ & 5 & $3.03 \%$ \\
\hline
\end{tabular}

(d) Pati-Salam

\begin{tabular}{lcc}
\hline Gauge group & $\begin{array}{c}\text { Number of unique } \\
\text { models }\end{array}$ & $\begin{array}{c}\text { Percentage of unique } \\
\text { models }\end{array}$ \\
\hline$S U(3)$ & 12 & $9.6 \%$ \\
$S U(5)$ & 8 & $6.4 \%$ \\
$S U(6)$ & 25 & $20 \%$ \\
$S U(8)$ & 29 & $23.2 \%$ \\
$S U(9)$ & 24 & $19.2 \%$ \\
$S U(10)$ & 15 & $12 \%$ \\
$S U(11)$ & 3 & $2.4 \%$ \\
$S U(12)$ & 7 & $5.6 \%$ \\
$S O(8)$ & 9 & $7.2 \%$ \\
$S O(10)$ & 11 & $8.8 \%$ \\
$S O(12)$ & 22 & $17.6 \%$ \\
$S O(14)$ & 19 & $15.2 \%$ \\
$S O(16)$ & 4 & $3.2 \%$ \\
$S O(20)$ & 2 & $1.6 \%$ \\
$E_{6}$ & 1 & $0.8 \%$ \\
$U(1)$ & 123 & $98.4 \%$ \\
\hline
\end{tabular}

groups and matter states. Only one model, generated by Table 9(a), in those discussed thus far exhibits full-gauge mirroring. However, the matter states are not mirrored. The particle content of that model is presented in Table 9(b).

The gauge groups are completely mirrored, and the matter representations are almost mirrored between one another. There is a state charged as a $\mathbf{1 6}$ under both $S O(16)$ groups and one charged as a $\mathbf{1 2 8}$ under one of the $S O(16)$ groups, but not the other. Thus, the matter is not mirrored. The potential for mirroring is clear from the basis vectors: $\bar{\psi}^{1, \ldots, 5}$ and $\bar{\eta}^{1,2,3}$ are mirrored with $\bar{\phi}^{1, \ldots, 8}$. There are also many models in which the observable and some of the hidden matter are mirrored, but include a shadow sector gauge group for which matter representations are not coupled.

These have been presented and discussed in [50].

\section{Conclusions}

Though there were many models containing GUTs in the data sets explored in this study, a vast majority of them do not contain any net chiral fermion generations. No three-generation models were found. These conclusions are summarized in Table 3.

While there were more models with GUT gauge groups in the NAHE variation $L 1 O 3$ extensions, none of them had any net chiral matter generations, implying that the added basis vector produces the barred and unbarred generations in even pairs, if at all. More complicated basis vector sets will need to be studied to determine if any NAHE variation-based quasirealistic models can be constructed.

The distribution of ST SUSYs across the subsets of GUT models was also examined. It was concluded that, as was the 
TABLE 9: A near mirrored, NAHE variation order-3 extension.

(a) Basis vector

\begin{tabular}{lcccccccccccccc}
\hline Sec $O$ & $\psi$ & $x^{12}$ & $x^{34}$ & $x^{56}$ & $\bar{\psi}^{1, \ldots, 5}$ & $\bar{\eta}^{1}$ & $\bar{\eta}^{2}$ & $\bar{\eta}^{3}$ & $\bar{\phi}$ & $y^{12} \|,{ }^{12}$ & $y^{34} \| \bar{y}^{34}$ & $y^{56} \| \bar{y}^{56}$ & $w^{1, \ldots, 6} \| \bar{w}^{1, \ldots, 6}$ \\
\hline$\vec{v}$ & 3 & 1 & 1 & 0 & 0 & $0, \ldots, 0$ & $2 / 3$ & $2 / 3$ & $2 / 3$ & $0, \ldots, 0,2 / 3,2 / 3,2 / 3$ & $0,0 \| 0,0$ & $1,1 \| 0,0$ & $1,1 \| 0,0$ & $0,0,0,0,0,0 \| 0,0,0,0,0,0$ \\
\hline$k_{\vec{v}, j}=(0,0,0,0,0)$ & & & & & & & & & &
\end{tabular}

(b) Particle content

\begin{tabular}{|c|c|c|c|c|c|c|c|c|}
\hline QTY & $S U(2)$ & $S U(2)$ & $S U(2)$ & $S U(2)$ & $S U(2)$ & $S U(2)$ & $S O(16)$ & $S O(16)$ \\
\hline 1 & 2 & 2 & 2 & 2 & 1 & 1 & 1 & 1 \\
\hline 1 & 2 & 2 & 2 & 1 & 1 & 2 & 1 & 1 \\
\hline 1 & 2 & 2 & 1 & 2 & 2 & 1 & 1 & 1 \\
\hline 1 & 2 & 2 & 1 & 1 & 2 & 2 & 1 & 1 \\
\hline 1 & 2 & 2 & 1 & 1 & 1 & 1 & 16 & 1 \\
\hline 1 & 2 & 1 & 2 & 2 & 2 & 1 & 1 & 1 \\
\hline 1 & 2 & 1 & 2 & 1 & 2 & 2 & 1 & 1 \\
\hline 1 & 2 & 1 & 2 & 1 & 1 & 1 & 16 & 1 \\
\hline 1 & 2 & 1 & 1 & 2 & 1 & 1 & 1 & 16 \\
\hline 1 & 2 & 1 & 1 & 1 & 2 & 1 & 16 & 1 \\
\hline 1 & 2 & 1 & 1 & 1 & 1 & 2 & 1 & 16 \\
\hline 1 & 1 & 2 & 2 & 2 & 2 & 1 & 1 & 1 \\
\hline 1 & 1 & 2 & 2 & 1 & 2 & 2 & 1 & 1 \\
\hline 1 & 1 & 2 & 2 & 1 & 1 & 1 & 16 & 1 \\
\hline 1 & 1 & 2 & 1 & 2 & 1 & 1 & 1 & 16 \\
\hline 1 & 1 & 2 & 1 & 1 & 2 & 1 & 16 & 1 \\
\hline 1 & 1 & 2 & 1 & 1 & 1 & 2 & 1 & 16 \\
\hline 1 & 1 & 1 & 2 & 2 & 1 & 1 & 1 & 16 \\
\hline 1 & 1 & 1 & 2 & 1 & 2 & 1 & 16 & 1 \\
\hline 1 & 1 & 1 & 2 & 1 & 1 & 2 & 1 & 16 \\
\hline 1 & 1 & 1 & 1 & 2 & 2 & 1 & 1 & 16 \\
\hline 1 & 1 & 1 & 1 & 2 & 1 & 2 & 16 & 1 \\
\hline 1 & 1 & 1 & 1 & 1 & 2 & 2 & 1 & 16 \\
\hline 1 & 1 & 1 & 1 & 1 & 1 & 1 & 128 & 1 \\
\hline 1 & 1 & 1 & 1 & 1 & 1 & 1 & 16 & 16 \\
\hline
\end{tabular}

case with the NAHE study, $E_{6}$ has a statistical coupling to enhanced ST SUSYs for order-3 models. Additionally, data sets in which all of the models contained at least one $U(1)$ factor with a GUT group had fewer models with $N=1 \mathrm{ST}$ SUSY.

Models with partial gauge group mirroring were also discussed, with a model presented that has complete gauge group mirroring. While a statistical search algorithm for finding quasi-mirrored models has not yet been completed, it will be used in future work to examine models with this property.

\section{Acknowledgment}

This work was supported by funding from Baylor University.

\section{References}

[1] R. Bousso and J. Polchinski, "Quantization of four-form fluxes and dynamical neutralization of the cosmological constant," The Journal of High Energy Physics, no. 06, article 006, 2000.

[2] S. K. Ashok and M. R. Douglas, "Counting flux vacua," Journal of High Energy Physics, no. 01, article 60, 2004.

[3] T. P. T. Dijkstra, L. R. Huiszoon, and A. N. Schellekens, "Supersymmetric standard model spectra from RCFT orientifolds," Nuclear Physics B, vol. 710, no. 1-2, pp. 3-57, 2005.

[4] R. Donagi, Y.-H. He, B. A. Ovrut, and R. Reinbacher, “The spectra of heterotic standard model vacua," Journal of High Energy Physics, no. 06, article 070, 2005.

[5] R. Valandro, Phenomenology from the landscape of string vacua," [Ph.D. thesis], http://arxiv.org/abs/0801.0584. 
[6] V. Balasubramanian, J. de Boer, and A. Naqvi, "Statistical predictions from anarchic field theory landscapes," Physics Letters $B$, vol. 682, pp. 476-483, 2010.

[7] O. Lebedev, H. P. Nilles, S. Ramos-Sánchez, M. Ratz, and P. K. S. Vaudrevange, "Heterotic mini-landscape (II): completing the search for MSSM vacua in a $Z_{6}$ orbifold," Physics Letters $B$, vol. 668, no. 4, pp. 331-335, 2008.

[8] F. Gmeiner and G. Honecker, "Millions of standard models on $Z_{6}{ }^{\prime}$ ?" Journal of High Energy Physics, no. 07, aticle 052, 2008.

[9] K. R. Dienes and M. Lennek, "Correlation classes on the landscape: to what extent is string theory predictive?" Physical Review D, vol. 80, no. 10, Article ID 106003, 12 pages.

[10] M. Gabella, Y.-H. He, and A. Lukas, "An abundance of heterotic vacua," Journal of High Energy Physics, no. 12, article 027, 2008.

[11] R. Donagi and K. Wendland, "On orbifolds and free fermion constructions," Journal of Geometry and Physics, vol. 59, no. 7, pp. 942-968, 2009.

[12] I. Antoniadis, C. P. Bachas, and C. Kounnas, "Four-dimensional superstrings," Nuclear Physics B, vol. 289, no. 1, pp. 87-108, 1987.

[13] I. Antoniadis and C. Bachas, " $4 \mathrm{~d}$ fermionic superstrings with arbitrary twists," Nuclear Physics B, vol. 298, no. 3, pp. 586-612, 1988.

[14] H. Kawai, D. C. Lewellen, and S.-H. H. Tye, "Construction of fermionic string models in four dimensions," Nuclear Physics B, vol. 288, no. 1, pp. 1-76, 1987.

[15] H. Kawai, D. C. Lewellen, J. A. Schwartz, and S.-H. H. Tye, “The spin structure construction of string models and multiloop modular invariance," Nuclear Physics B, vol. 299, no. 3, pp. 431470, 1988.

[16] G. Cleaver, A. Faraggi, D. Nanopoulos, and J. Walker, "A minimal superstring standard model II: a Phenomenological Study," Nuclear Physics B, vol. 593, no. 3, pp. 471-504, 2001.

[17] J. Lopez, D. Nanopoulos, and K. Yuan, "The search for a realistic flipped SU(5) string model," Nuclear Physics B, vol. 399, no. 2-3, pp. 654-688, 1993.

[18] A. E. Faraggi, D. V. Nanopoulos, and K. Yuan, "A standard-like model in the four-dimensional free fermionic string formulation," Nuclear Physics B, vol. 335, no. 2, pp. 347-362, 1990.

[19] A. E. Faraggi, "Construction of realistic standard-like models in the free fermionic superstring formulation," Nuclear Physics B, vol. 387, no. 2, pp. 239-262, 1992.

[20] I. Antoniadis, G. K. Leontaris, and J. Rizos, "A three-generation $\mathrm{SU}(4)$ x O(4) string model," Physics Letters B, vol. 245, no. 2, pp. 161-168, 1990.

[21] G. K. Leontaris and J. Rizos, " $N=1$ supersymmetric $S U(4) \times$ $S U(2)_{L} \times S U(2)_{R}$ effective theory from the weakly coupled heterotic superstring," Nuclear Physics B, vol. 554, no. 1-2, pp. 3-49, 1999.

[22] A. E. Faraggi, "A new standard-like model in the four dimensional free fermionic string formulation," Physics Letters B, vol. 278, no. 1-2, pp. 131-139, 1992.

[23] A. E. Faraggi, "Aspects of non-renormalizable terms in a superstring derived standard-like model," Nuclear Physics B, vol. 403, no. 1-2, pp. 101-121, 1993.

[24] A. E. Faraggi, "Generation mass hierarchy in superstring derived models," Nuclear Physics B, vol. 407, no. 1, pp. 57-72, 1993.

[25] A. E. Faraggi, "Hierarchical top-bottom mass relation in a superstring derived standard-like model," Physics Letters B, vol. 274, no. 1, pp. 47-52, 1992.
[26] A. E. Faraggi, "Yukawa couplings in superstring-derived standardlike models," Physical Review D, vol. 47, no. 11, pp. 50215028, 1993.

[27] A. E. Faraggi, "Top quark mass prediction in superstring derived standard-like models," Physics Letters B, vol. 377, no. 13, pp. 43-47, 1996.

[28] A. E. Faraggi, "Calculating fermion masses in superstring derived standard-like models," Nuclear Physics B, vol. 487, no. 1-2, pp. 55-92, 1997.

[29] G. B. Cleaver, "Advances in old-fashioned heterotic string model building," Nuclear Physics B, vol. 62, no. 1-3, pp. 161-170, 1998.

[30] G. B. Cleaver and A. E. Faraggi, "On the anomalous $U(1)$ in free fermionic superstring models," International Journal of Modern Physics A, vol. 14, no. 15, pp. 2335-2356, 1999.

[31] G. Cleaver, M. Cvetič, J. R. Espinosa, L. Everett, and P. Langacker, "Classification of flat directions in perturbative heterotic superstring vacua with anomalousU(1)," Nuclear Physics B, vol. 525, no. 1-2, pp. 3-26, 1998.

[32] G. Cleaver, M. Cvetič, J. R. Espinosa, L. Everett, and P. Langacker, "Flat directions in three-generation free-fermionic string models," Nuclear Physics B, vol. 545, no. 1-3, pp. 47-97, 1999.

[33] G. Cleaver, M. Cvetič, J. Espinosa, L. Everett, P. Langacker, and J. Wang, "Physics implications of flat directions in free fermionic superstring models I. Mass spectrum and couplings," Physical Review D, vol. 59, no. 5, Article ID 055005, 21 pages, 1999.

[34] G. Cleaver, M. Cvetič, J. Espinosa, L. Everett, P. Langacker, and J. Wang, "Physics implications of flat directions in free fermionic superstring modelsII. Renormalization group analysis," Physical Review D, vol. 59, no. 11, Article ID 115003, 18 pages.

[35] G. B. Cleaver, "Quark masses and flat directions in string models," in Proceeding of the 4th workshop on QCD, Paris, France, June 1998, http://arxiv.org/abs/hep-ph/9812262.

[36] G. B. Cleaver, A. E. Faraggi, and D. V. Nanopoulos, "String derived MSSM and M-theory unification," Physics Letters B, vol. 455, no. 1-4, pp. 135-146, 1999.

[37] G. B. Cleaver, A. E. Faraggi, and D. V. Nanopoulos, "A minimal superstring standard model. I. Flat directions," International Journal of Modern Physics A, vol. 16, no. 3, pp. 425-482, 2001.

[38] G. Cleaver, A. Faraggi, D. Nanopoulos, and J. Walke, "Phenomenological study of a minimal superstring standard model," Nuclear Physics B, vol. 593, no. 3, pp. 471-504, 2001.

[39] G. B. Cleaver, "M-fluences on string model building," submitted, http://arxiv.org/abs/hep-ph/9901203.

[40] G. B. Cleaver, A. E. Faraggi, D. V. Nanopoulos, and J. W. Walker, "Non-Abelian flat directions in a minimal superstring standard model," Modern Physics Letters A, vol. 15, no. 18, p. 1191, 2000.

[41] G. B. Cleaver, A. E. Faraggi, and C. Savage, "Left-right symmetric heterotic-string derived models," Physical Review D, vol. 63, no. 6, Article ID 066001, 22 pages, 2001.

[42] G. B. Cleaver, A. E. Faraggi, D. V. Nanopoulos, and J. W. Walker, "Phenomenology of non-abelian flat directions in a minimal superstring standard model," Nuclear Physics B, vol. 620, no. 1-2, pp. 259-289, 2002.

[43] G. B. Cleaver, D. J. Clements, and A. E. Faraggi, "Flat directions in left-right symmetric string derived models," Physical Review D, vol. 65, no. 10, Article ID 106003, 11 pages, 2002.

[44] G. B. Cleaver, A. E. Faraggi, and S. E. M. Nooij, "NAHE-based string models with $S U(4) \times S U(2) \times U(1) S O(10)$," Nuclear Physics $B$, vol. 672 , no. 1-2, pp. 64-86, 2003. 
[45] G. Cleaver, "Parameter space investigations of free fermionic heterotic models," Proceedings of the 1st International conference on string phenomenology, Oxford, UK, July 2002, http:// arxiv.org/abs/hep-ph/0210093.

[46] G. Cleaver, V. Desai, H. Hanson, J. Perkins, D. Robbins, and S. Shields, "Possibility of optical unification in heterotic strings," Physical Review D, vol. 67, no. 2, Article ID 026009, 8 pages, 2003.

[47] J. Perkins, B. Dundee, R. Obousy et al., "Stringent phenomenological investigation into heterotic string optical unification," Physical Review D, vol. 75, no. 2, Article ID 026007, 2007.

[48] J. Perkins, B. Dundee, R. Obousy et al., "Stringent phenomenological investigation into heterotic string optical unification," Physical Review D, vol. 75, no. 2, Article ID 026007, 17 pages, 2007.

[49] G. Cleaver, A. E. Faraggi, E. Manno, and C. Timirgaziu, "Quasi-realistic heterotic-string models with vanishing oneloop cosmological constant and perturbatively broken supersymmetry?" Physical Review D, vol. 78, no. 4, Article ID 046009, 2008.

[50] J. Greenwald, D. Moore, K. Pechan, T. Renner, T. Ali, and G. Cleaver, "Note on a NAHE Variation," Nuclear Physics B, vol. 850, no. 3, pp. 445-462, 2011.

[51] A. E. Faraggi, "MSHSM-minimal standard eeterotic string models," Fortschritte der Physik, vol. 58, no. 7-9, pp. 733-737, 2010.

[52] B. Gato-Rivera and A. N. Schellekens, "Asymmetric Gepner models (revisited)," Nuclear Physics B, vol. 841, no. 1-2, pp. 100129, 2010.

[53] B. Gato-Rivera and A. N. Schellekens, "Asymmetric Gepner models II. Heterotic weight lifting," Nuclear Physics B, vol. 846, no. 3, pp. 429-468, 2011.

[54] B. Gato-Rivera and A. N. Schellekens, "Asymmetric Gepner models III. B-L lifting," Nuclear Physics B, vol. 847, no. 3, pp. 532-548, 2011.

[55] M. Maio and A. N. Schellekens, "Permutation orbifolds of heterotic Gepner models," Nuclear Physics B, vol. 848, no. 3, pp. 594-628, 2011.

[56] B. Assel, K. Christodoulides, A. E. Faraggi, C. Kounnas, and J. Rizos, "Classification of heterotic Pati-Salam models," Nuclear Physics B, vol. 844, no. 3, pp. 365-396, 2011.

[57] G. Cleaver, A. E. Faraggi, G. Greenwald et al., "Investigation of quasi-realistic heterotic string models with reduced Higgs spectrum," The European Physical Journal C, vol. 71, p. 1842, 2011.

[58] T. Renner, J. Greenwald, D. Moore, and G. Cleaver, "Initial systematic investigations of the landscape of low layer NAHE extensions," The European Physical Journal C, vol. 72, p. 2167, 2012.

[59] R. N. Mohapatra and L. Vigdor Teplitz, "Structures in the mirror universe," The Astrophysical Journal, vol. 478, no. 1, pp. 29-38, 1997.

[60] R. Mohapatra and V. Teplitz, "Mirror matter MACHOs," Physics Letters B, vol. 462, no. 3-4, pp. 302-309, 1999.

[61] R. Mohapatra and V. Teplitz, "Mirror dark matter," in Proceedings of the 14th International symposium on sources and Detection of Dark Matter in the Universe, Marina del Rey, Calif, USA, February 2004, http://arxiv.org/abs/astro-ph/0004046.

[62] R. Mohapatra, S. Nussinov, and V. Teplitz, "Mirror matter as self-interacting dark matter," Physical Review D, vol. 66, no. 6, Article ID 063002, 2002. 

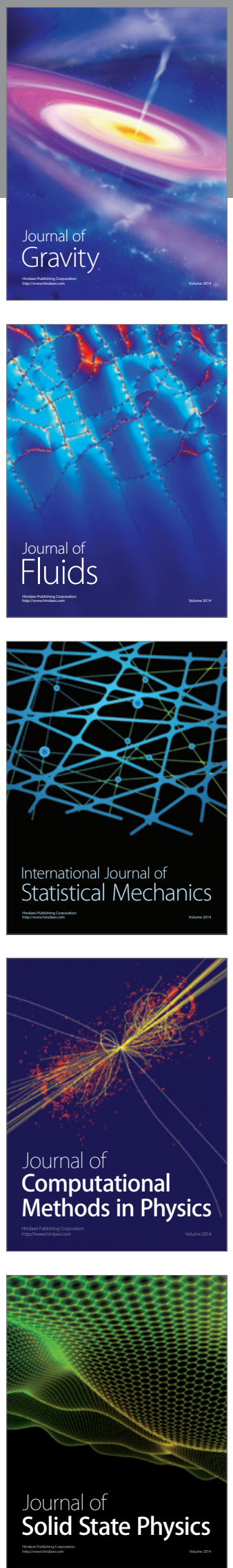

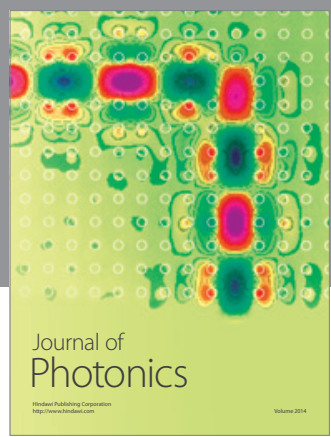

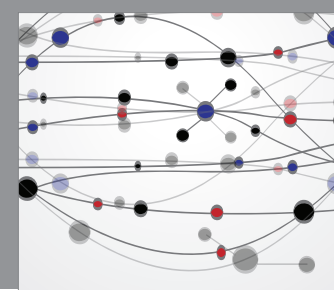

The Scientific World Journal

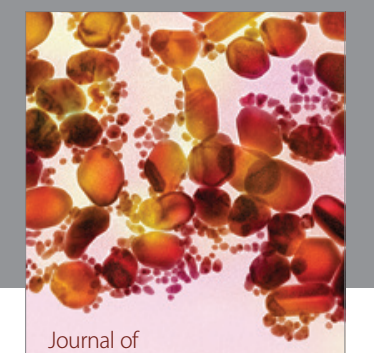

Soft Matter
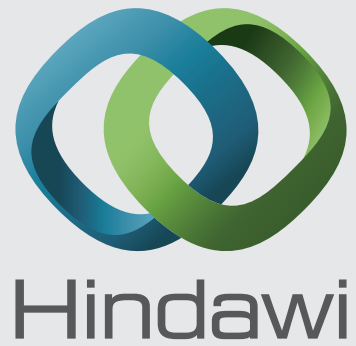

Submit your manuscripts at

http://www.hindawi.com
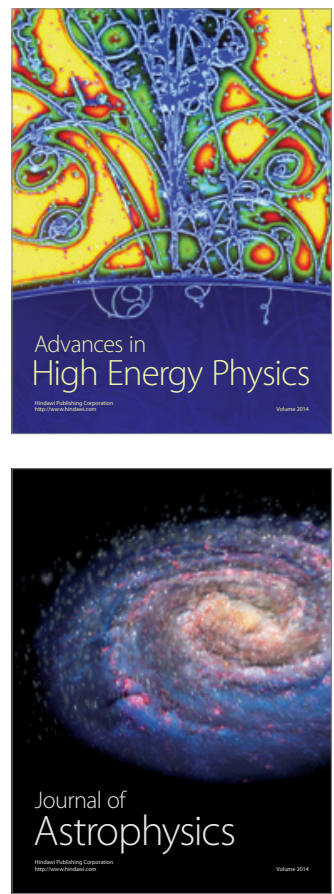
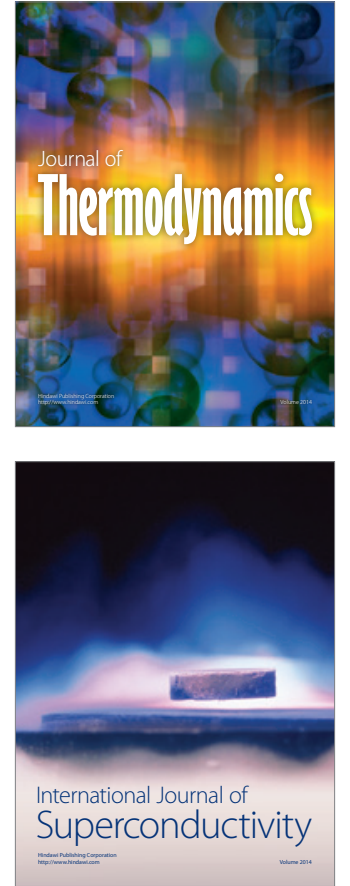
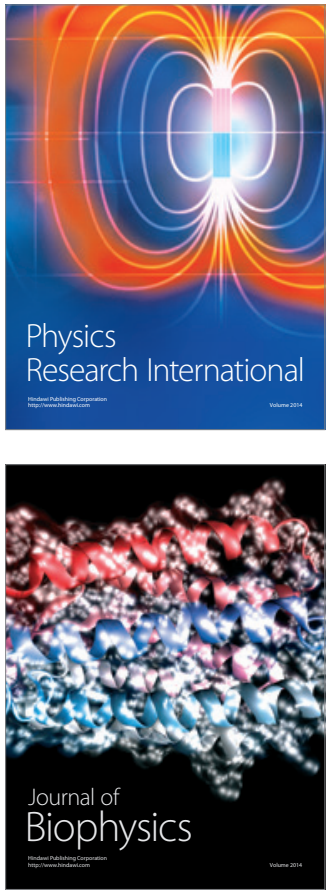
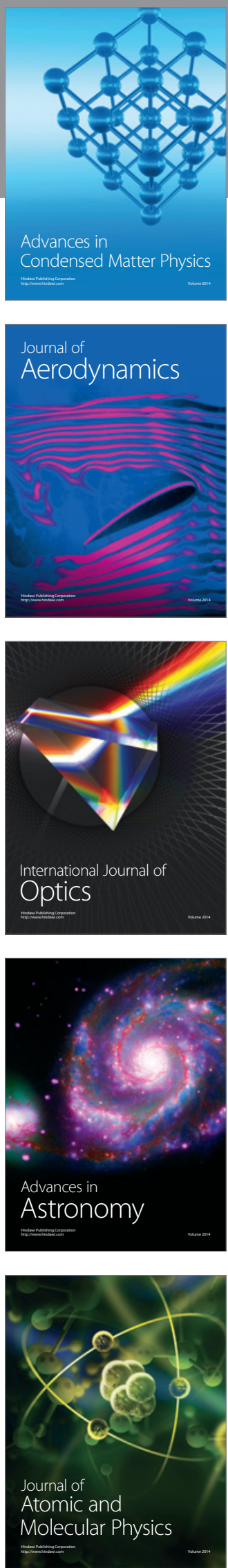